\title{
Trust Based Consensus Model for Social Network in an Incomplete Linguistic Information Context ${ }^{\text {th }}$
}

\author{
Jian $\mathrm{Wu}^{\mathrm{a}, \mathrm{b}}$, Francisco Chiclana ${ }^{\mathrm{b}}$, Enrique Herrera-Viedma ${ }^{\mathrm{c}}$ \\ ${ }^{a}$ School of Economics and Management, Zhejiang Normal University, Jinhua, Zhejiang, China \\ ${ }^{b}$ Centre for Computational Intelligence, Faculty of Technology, De Montfort University, Leicester, UK \\ ${ }^{c}$ Department of Computer Science and Artificial Intelligence, University of Granada, Granada, Spain
}

\begin{abstract}
A theoretical framework to consensus building within a networked social group is put forward. This article investigates a trust based estimation and aggregation methods as part of a visual consensus model for multiple criteria group decision making with incomplete linguistic information. A novel trust propagation method is proposed to derive trust relationship from an incomplete connected trust network and the trust score induced order weighted averaging operator is presented to aggregate the orthopairs of trust/distrust values obtained from different trust paths. Then, the concept of relative trust score is defined, whose use is twofold: (1) to estimate the unknown preference values; and (2) as a reliable source to determine experts' weights. A visual feedback process is developed to provide experts with graphical representations of their consensus status within the group as well as to identify the alternatives and preference values that should be reconsidered for changing in the subsequent consensus round. The feedback process also includes a recommendation mechanism to provide advice to those experts that are identified as contributing less to consensus on how to change their identified preference values. It is proved that the implementation of the visual feedback mechanism guarantees the convergence of the consensus reaching process.
\end{abstract}

Keywords: Social network, Multiple criteria group decision making, Trust propagation, Trust aggregation, Visual feedback, Incomplete linguistic information

\section{Introduction}

Social network analysis $[25,47,50]$ studies the relationships between social entities like members of a group, corporations or nations. Of particular interest, and the focus of this paper, is to investigate consensus building between a group of experts connected via a network in which they explicitly express opinions in the form of trust and distrust statements, which are referred herein as trust network [48] and orthopairs of trust/distrust values [16], respectively. By analysing trust relationships between the

${ }^{\text {th }}$ Cite as: Jian Wu, Francisco Chiclana, Enrique Herrera-Viedma: 'Trust Based Consensus Model for Social Network in an Incomplete Linguistic Information Context.' Applied Soft Computing. In press, 2015. doi: 110.1016/j.asoc.2015.02.023

Email addresses: jyajian@163.com (Jian Wu), chiclana@dmu.ac.uk (Francisco Chiclana), viedma@decsai.ugr.es (Enrique Herrera-Viedma) 
networked experts, the concept of trust score and knowledge deficit are defined and used to propose an order relation on the set of orthopairs of trust/distrust values, which will be used to distinguish the most trusted expert from the group and, ultimately, to drive the aggregation of the individual opinions in order to arrive at a group consensual decision making solution.

In a general multiple criteria group decision making (MCGDM), a group of experts express preference values on alternatives under multiple criteria and interact to derive a common solution [35]. Experts usually come from multiple organisations and/or may have different backgrounds and knowledge on the decision making problem faced. Over the past decades, a large number of researchers have been attracted into this field [8-10, 17, 29-31, 34, 65]. These proposed models have been developed under the assumption that the preference values on alternatives under multiple criteria are completely expressed by experts. However, this assumption may not be completely realistic due to lack of in-depth knowledge of the problem domain by all or some of experts [32, 45]. Thus, a key issue that needs to be addressed in this type of decision making environment is that of "estimating unknown preference values". There exist algorithms available to estimate unknown preference values in decision making based on the notion of consistency but not in trust $[1-4,7,12,13,27$, 41], which is a new key knowledge that is possible to find in social network [33, 46]. An objective of this paper is to develop a social trust based estimation method for MCGDM with incomplete preferences. On the other hand, another key issue in this type of decision making problem is how to reach consensus to derive the decision solution $[6,28]$. The interactive consensus model is regarded as an effective method to reach satisfactory consensus level because it implements a feedback mechanism to advice experts on how to change their preferences $[5,19,23,24,54,57,64]$. The known interactive consensus models force experts to change their preference values when consensus is below a threshold value. However, this may conflict with decision making in real practice because it is up to the experts to implement or not the given recommendations $[22,53]$. Additionally, these consensus models have the limitation that there is no visual representation to help them analyse their consensus position within the group.

The aim of this paper is to present a new trust based consensus model for social network in a 2-tuple linguistic context [20,21, 26, 38] under incomplete information. In this model, a policy allowing experts to revisit their evaluations using appropriate and meaningful consensus information representation within the social network framework is implemented. Firstly, a novel social trust propagation method to derive unknown information associated to an expert using trusted third partners (TTPs) is proposed. The trust score induced order weighted averaging (TS-IOWA) operator is developed to aggregate the orthopairs of trust/distrust values obtained from different trust paths. Secondly, a novel visual feedback process for MCGDM is designed to provide experts with: (1) visual representations of their consensus status within the group; and (2) individual advice on how to change preference values. Additionally, visual simulation of future consensus status is generated to support experts in revisiting their evaluations and make changes to achieve a higher level of consensus. When this visual feedback 
mechanism is used to guide the consensus reaching process then its convergence is guaranteed within the social network.

The rest of paper is set out as follows: Section 2 introduces the trust network and an order relation of orthopairs of trust/distrust values, as well as the novel trust propagation and trust aggregation operators. In Section 3, the concept of relative trust score (RTS) and average trust degree (ATD) are defined. The first degree is used to estimate the unknown 2-tuple linguistic preference values, while the second one is used to aggregate the individual 2-tuple linguistic preference relations. Section 4 presents a new visual consensus model for social network that integrates visual representations of experts' consensus status within the group, the identification of experts and preference values that contribute less to consensus, individual advice on how to change preference values and visual simulation of future consensus status. The convergence of the consensus reaching process is also proved when this visual feedback mechanism is used to guide it. An analysis of the proposed visual consensus model with respect to existing consensus models in literature is given in Section 5. Finally, conclusions are drawn in Section 6.

\section{Trust Propagation and Aggregation in Social Network}

Social Network Analysis (SNA) [25, 47, 50] studies the relationships between social entities like members of a group, corporations or nations. Therefore, it enables us to examine their structural and locational properties including centrality, prestige, structural balance, trust relationship etc. There are three notational schemes in SNA analysis: set of actors, the relations themselves, and the actor criteria (see Table 1). As a consequence, we can refer to important network concepts in a unified manner.

- Graph theoretic: in which the network is viewed as a graph consisting of nodes joined by lines.

- Algebraic: this notation presents the advantage that allow us to distinguish several distinct relations and represent combinations of relations.

- Sociometric: in which relational data are often presented in two-ways matrices called sociomatrix.

The above sociomatrix is a binary or crisp relation. However, in many situations, it may not be suitable to represent the relation in a crisp way because it is not clear cut defined. Notice that in real life too, trust is often interpreted as a gradual concept as humans do not just reason in terms of 'trusting' and 'not trusting', but rather trusting someone 'very much' or 'more or less' [18]. Victor et al. in [48] introduce the following adapted bilattice structure based on the use of orthopairs of trust/distrust values as follows: 
Table 1: Different notations in Social Network Analysis

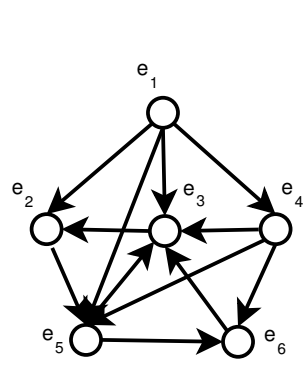

Graph

$\begin{array}{ll}E_{1} R E_{2} & E_{4} R E_{3} \\ E_{1} R E_{3} & E_{4} R E_{5} \\ E_{1} R E_{4} & E_{4} R E_{6} \\ E_{1} R E_{5} & E_{5} R E_{3} \\ E_{2} R E_{5} & E_{5} R E_{6} \\ E_{3} R E_{2} & E_{6} R E_{3}\end{array} \quad A=\left(\begin{array}{cccccc}0 & 1 & 1 & 1 & 1 & 0 \\ 0 & 0 & 0 & 0 & 1 & 0 \\ 0 & 1 & 0 & 0 & 0 & 0 \\ 0 & 0 & 1 & 0 & 1 & 1 \\ 0 & 0 & 1 & 0 & 0 & 1 \\ 0 & 0 & 1 & 0 & 0 & 0\end{array}\right)$

Sociometric

Definition 1. The set of orthopairs of trust/distrust values $\left(B L^{\square}\right)$ can be endowed with a bilattice structure with the following trust ordering $\left(\leq_{t}\right)$, knowledge ordering $\left(\leq_{k}\right)$, and negation operator $(\neg)$ :

$$
\begin{aligned}
& B L^{\square}=\left([0,1]^{2}, \leq_{t}, \leq_{k}, \neg\right) \\
& \left(t_{1}, d_{1}\right) \leq_{t}\left(t_{2}, d_{2}\right) \text { iff } t_{1} \leq t_{2} \text { and } d_{1} \geq d_{2} \\
& \left(t_{1}, d_{1}\right) \leq_{k}\left(t_{2}, d_{2}\right) \text { iff } t_{1} \leq t_{2} \text { and } d_{1} \leq d_{2} \\
& \neg\left(t_{1}, d_{1}\right)=\left(d_{1}, t_{1}\right)
\end{aligned}
$$

As stated by Victor et al. in [48], it is clear that "the lattice $\left([0,1]^{2}, \leq_{t}\right)$ orders the [orthopairs of trust/distrust values] going from complete distrust $(0,1)$ to complete trust $(1,0)$, [while] the $\left([0,1]^{2}, \leq_{k}\right)$ evaluates the amount of available trust evidence, ranging from [...] incomplete information $\left[t_{1}+d_{1}<1\right]$ to $[\ldots]$ inconsistent or contradictory information $\left[t_{1}+d_{1}>1\right]$ ]". Thus, two orthopairs of trust/distrust values with same trust values and different distrust values will be placed in reverse order by the trust ordering $\leq_{t}$ and the knowledge ordering $\leq_{k}$, and thus to avoid this outcome Victor et al.'s ordering approach will not be used. Having said this, it is noticed here that the methodology used in the case of intuitionistic fuzzy sets $[52,55]$ for the concepts of score and accuracy of an intuititionistic value can be applied in this context to define the following concepts of trust score and knowledge deficit so that a two steps complete ordering on the set of orthopairs of trust/distrust values can be derived [48]:

Definition 2 (Trust Score and Knowledge deficit). The trust score and knowledge deficit associated to an orthopair of trust/distrust values $\left(t_{1}, d_{1}\right)$ are:

$$
\begin{gathered}
T S\left(t_{1}, d_{1}\right)=t_{1}-d_{1} \\
K D\left(t_{1}, d_{1}\right)=\left|1-t_{1}-d_{1}\right| .
\end{gathered}
$$

Following Victor et al. in [48], we say that orthopairs of trust/distrust values $\left(t_{1}, d_{1}\right)$ for which $K D\left(t_{1}, d_{1}\right)=0$, i.e., $t_{1}+d_{1}=1$, have perfect knowledge (i.e., complete trust state), while all others will have a deficit in knowledge. The combination of both trust score and knowledge deficit can be used to propose the following order relation for the set of orthopairs of trust/distrust values: 
Definition 3. Let $\left(t_{1}, d_{1}\right)$ and $\left(t_{2}, d_{2}\right)$ be orthopairs of trust/distrust values, $T S_{1}=t_{1}-d_{1}$ and $T S_{2}=t_{2}-d_{2}$ their associated trust scores, and $K D\left(t_{1}, d_{1}\right)=\left|1-t_{1}-d_{1}\right|$ and $K D\left(t_{2}, d_{2}\right)=\left|1-t_{2}-d_{2}\right|$ their associated knowledge deficits, respectively. We have that

1. If $T S_{1}<T S_{2}$, then $\left(t_{1}, d_{1}\right)$ is smaller than $\left(t_{2}, d_{2}\right)$, denoted by $\left(t_{1}, d_{1}\right)<\left(t_{2}, d_{2}\right)$;

2. If $T S_{1}>T S_{2}$, then $\left(t_{1}, d_{1}\right)$ is greater than $\left(t_{2}, d_{2}\right)$, denoted by $\left(t_{1}, d_{1}\right)>\left(t_{2}, d_{2}\right)$;

3. If $T S_{1}=T S_{2}$, then:

(a) if $K D\left(t_{1}, d_{1}\right)<K D\left(t_{2}, d_{2}\right)$, then $\left(t_{1}, d_{1}\right)$ is greater than $\left(t_{2}, d_{2}\right)$, denoted by $\left(t_{1}, d_{1}\right)>$ $\left(t_{2}, d_{2}\right)$;

(b) if $K D\left(t_{1}, d_{1}\right)>K D\left(t_{2}, d_{2}\right)$, then $\left(t_{1}, d_{1}\right)$ is smaller than $\left(t_{2}, d_{2}\right)$, denoted by $\left(t_{1}, d_{1}\right)<$ $\left(t_{2}, d_{2}\right) ;$

(c) if $K D\left(t_{1}, d_{1}\right)=K D\left(t_{2}, d_{2}\right)$, then $\left(t_{1}, d_{1}\right)$ is equal to $\left(t_{2}, d_{2}\right)$, denoted by $\left(t_{1}, d_{1}\right)=\left(t_{2}, d_{2}\right)$.

Thus, when comparing two orthopairs of trust/distrust values, the one with higher trust score is ordered first, and in case of equal trust scores, the lower knowledge deficit prevails. We can utilise the order relation of orthopairs of trust/distrust values to distinguish the most trusted expert from a group or path in a trust network, which is useful in fusing individual opinions because it can be used to induce the ordering of an OWA based aggregation of the decision matrix values. This will be elaborated later in the paper.

\subsection{Trust propagation}

In a trust network, some experts may be typically unknown to a specific expert, which means they are not able to give the orthopairs of trust/distrust values on him/her directly. However, we still can derive some information on whether or not an unknown expert can be trusted by using indirect paths of trusted third parties (TTPs). This is illustrated in Fig. 1 where three experts are illustrated in which there is no direct orthopair of trust/distrust values between expert $E_{1}$ and $E_{3}$, although an indirect orthopair of trust/distrust values between expert $E_{1}$ and $E_{3}$ can be obtained by propagating the corresponding values of the path via expert $E_{2}$.

The above scenario was investigated by Victor et al. in [48], where the following propagation operator was proposed:

$$
P\left(\left(t_{1}, d_{1}\right),\left(t_{2}, d_{2}\right)\right)=\left(T\left(t_{1}, t_{2}\right), T\left(t_{1}, d_{2}\right)\right)
$$

with $T$ being a t-norm as illustrated in Fig. 2. This operator reflects the expected behaviour:

- expert $E_{1}$ trusts $E_{3}$ when $E_{1}$ trusts $E_{2}$ and $E_{2}$ trusts $E_{3}: P\left(\left(1, d_{1}\right),\left(1, d_{2}\right)\right)=\left(T(1,1), T\left(1, d_{2}\right)\right)=$ $\left(1, d_{2}\right)$. 


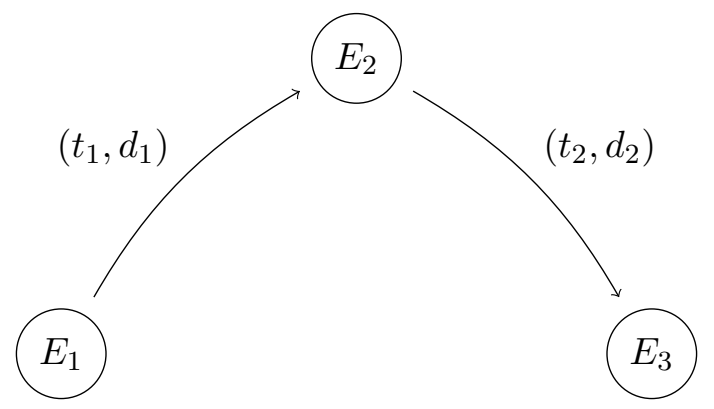

Figure 1: No direct orthopair of trust/distrust values between $E_{1}$ and $E_{3}$

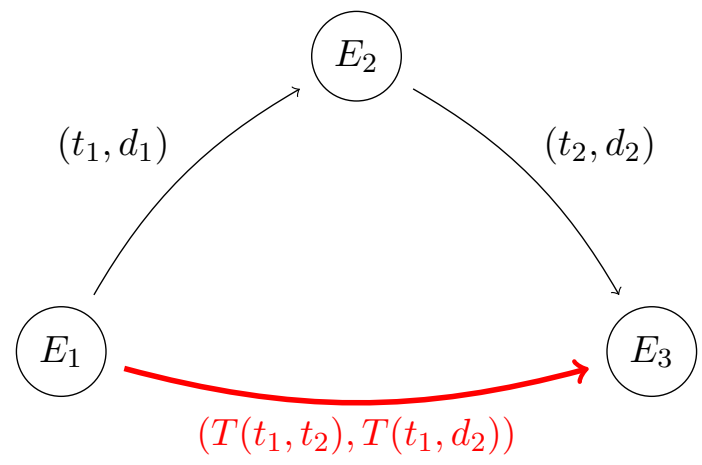

Figure 2: Trust propagation of orthopairs of trust/distrust values between $E_{1}$ and $E_{3}$ via $E_{2}$

- expert $E_{1}$ distrusts $E_{3}$ when $E_{2}$ distrusts $E_{3}$ and $E_{1}$ trusts $E_{2}: P\left(\left(1, d_{1}\right),\left(t_{2}, 1\right)\right)=\left(\left(T\left(1, t_{2}\right), T(1,1)\right)=\right.$ $\left(t_{2}, 1\right)$.

Recall that Victor et al. in [49] interpret the orthopair of trust/distrust values $(0,0)$ as representing complete ignorance. Obviously, when a trust path happens to contain an ignorance orthopair of trust/distrust values then the propagation operator output will also be an ignorance orthopair of trust/distrust values. Thus, an ignorance case will be treated here as an incomplete entry [2] rather than be modelled with the $(0,0)$ orthopair of trust/distrust values.

Notice that associativity of t-norm $T$ implies associativity of propagation operator $P$ :

$$
\begin{aligned}
P\left(P\left(\left(t_{1}, d_{1}\right),\left(t_{2}, d_{2}\right)\right),\left(t_{3}, d_{3}\right)\right) & =P\left(\left(T\left(t_{1}, t_{2}\right), T\left(t_{1}, d_{2}\right)\right),\left(t_{3}, d_{3}\right)\right) \\
& =\left(T\left(T\left(t_{1}, t_{2}\right), t_{3}\right), T\left(T\left(t_{1}, t_{2}\right), d_{3}\right)\right) \\
& =\left(T\left(t_{1}, T\left(t_{2}, t_{3}\right)\right), T\left(t_{1}, T\left(t_{2}, d_{3}\right)\right)\right) \\
& =P\left(\left(t_{1}, d_{1}\right),\left(T\left(t_{2}, t_{3}\right), T\left(t_{2}, d_{3}\right)\right)\right)=P\left(\left(t_{1}, d_{1}\right), P\left(\left(t_{2}, d_{2}\right),\left(t_{3}, d_{3}\right)\right)\right)
\end{aligned}
$$

Thus, an incomplete trust path as the one illustrated in Fig. 3 can be completed using the associativity property of $P$, i.e.

$$
P\left(\left(t_{1}, d_{1}\right),\left(t_{2}, d_{2}\right),\left(t_{3}, d_{3}\right)\right)=\left(T\left(t_{1}, t_{2}, t_{3}\right), T\left(t_{1}, t_{2}, d_{3}\right)\right)
$$

as illustrated in Fig. 4.

Expression (2) can be extended to an arbitrary number of experts $n(\geq 3)$ as follows:

$$
P\left(\left(t_{1}, d_{1}\right),\left(t_{2}, d_{2}\right), \ldots,\left(t_{n}, d_{n}\right)\right)=\left(T\left(t_{1}, t_{2}, \ldots, t_{n}\right), T\left(t_{1}, t_{2}, \ldots, t_{n-1}, d_{n}\right)\right)
$$




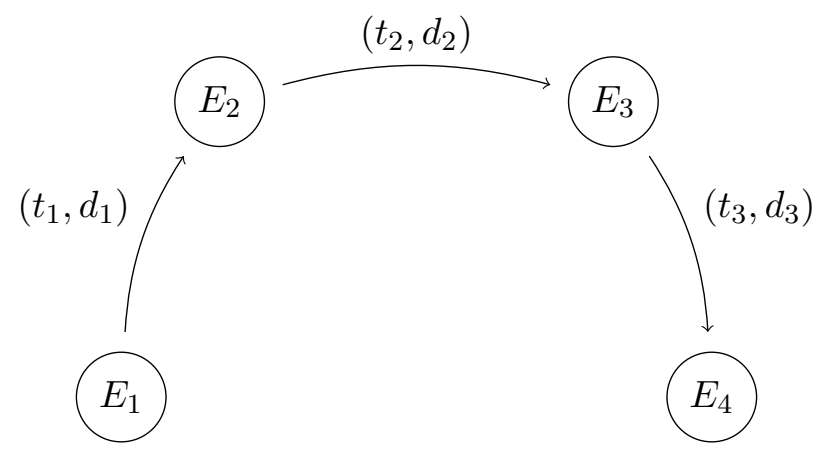

Figure 3: No direct orthopair of trust/distrust values between $E_{1}$ and $E_{4}$

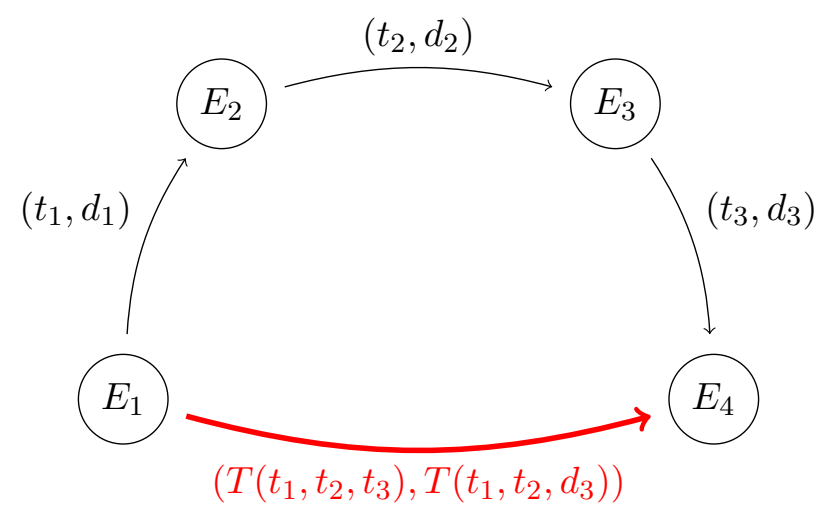

Figure 4: Trust propagation of orthopairs of trust/distrust values between $E_{1}$ and $E_{4}$ via trust path $E_{1} \rightarrow E_{2} \rightarrow E_{3} \rightarrow E_{4}$

To prove (3), induction on $n$ is applied:

1. Basis: $n=3$. This is the associativity property (2) already proved to be true.

2. Induction hypothesis: Let's assume that (3) is true for $n=k$ and prove that it is also true when $n=k+1$. Applying associativity of $P$ we have:

$$
P\left(\left(t_{1}, d_{1}\right),\left(t_{2}, d_{2}\right), \ldots,\left(t_{k+1}, d_{k+1}\right)\right)=P\left(P\left(\left(t_{1}, d_{1}\right),\left(t_{2}, d_{2}\right), \ldots,\left(t_{k}, d_{k}\right)\right),\left(t_{k+1}, d_{k+1}\right)\right)
$$

Applying that (3) is true for $n=k$ we have:

$$
P\left(\left(t_{1}, d_{1}\right),\left(t_{2}, d_{2}\right), \ldots,\left(t_{k+1}, d_{k+1}\right)\right)=P\left(\left(T\left(t_{1}, t_{2}, \ldots, t_{k}\right), T\left(t_{1}, t_{2}, \ldots, t_{k-1}, d_{k}\right)\right),\left(t_{k+1}, d_{k+1}\right)\right)
$$

Definition of $P$ is applied to obtain:

$$
P\left(\left(t_{1}, d_{1}\right),\left(t_{2}, d_{2}\right), \ldots,\left(t_{k+1}, d_{k+1}\right)\right)=\left(T\left(T\left(t_{1}, t_{2}, \ldots, t_{k}\right), t_{k+1}\right), T\left(T\left(t_{1}, t_{2}, \ldots, t_{k}\right), d_{k+1}\right)\right)
$$

Applying associativity of t-norm $T$ yields:

$$
P\left(\left(t_{1}, d_{1}\right),\left(t_{2}, d_{2}\right), \ldots,\left(t_{k+1}, d_{k+1}\right)\right)=\left(T\left(t_{1}, t_{2}, \ldots, t_{k+1}\right), T\left(t_{1}, t_{2}, \ldots, t_{k}, d_{k+1}\right)\right)
$$


In this paper, and for illustration purposes, we will be making use of the product t-norm $T(x, y)=x \cdot y$ and therefore

$$
P\left(\left(t_{1}, d_{1}\right),\left(t_{2}, d_{2}\right), \ldots,\left(t_{n}, d_{n}\right)\right)=\left(\prod_{i=1}^{n} t_{i},\left(\prod_{i=1}^{n-1} t_{i}\right) \cdot d_{n}\right)
$$

\subsection{Trust aggregation}

A crucial step in any group decision making (GDM) process is the aggregation of individual opinions with the aim of achieving a "fair" representation of each individual within the group. In 1988, Yager introduced an aggregation technique based on the order weighted averaging (OWA) scheme [58]. Since then, OWA based aggregation strategies have been widely investigated [15, 37, 43, 51, 61, 66].

Definition 4 (OWA Operator). An OWA operator of dimension $n$ is a mapping $\mathrm{OWA}_{W}: \mathbb{R}^{n} \rightarrow \mathbb{R}$, which has associated set of weights $W=\left(w_{1}, \cdots, w_{n}\right)^{T}$ to it, so that $w_{i} \in[0,1]$ (positive) and $\sum_{i=1}^{n} w_{i}=1$ (normalised),

$$
\mathrm{OWA}_{W}\left(a_{1}, \cdots, a_{n}\right)=\sum_{i=1}^{n} w_{i} a_{\sigma(i)}
$$

where $\sigma:\{1, \cdots, n\} \rightarrow\{1, \cdots, n\}$ is a permutation function such that $a_{\sigma(i)}$ is the $\mathrm{i}$-th highest value in the set $\left\{a_{1}, \cdots, a_{n}: a_{\sigma(i)} \geq a_{\sigma(i-1)}\right\}$.

The aggregation of orthopairs of trust/distrust values was proposed to be carried out by Victor et al. [49] using the following OWA based aggregation operator:

Definition 5 (K-OWA Operator). The K-OWA operator is the mapping K-OWA $W_{T}, W_{D}: B L^{\square^{n}} \rightarrow$ $B L^{\square}$,

$$
\left.\mathrm{K}-\mathrm{OWA}_{W_{T}, W_{D}}\left(\left(t_{1}, d_{1}\right), \ldots,\left(t_{n}, d_{n}\right)\right)\right)=\left(O W A_{W_{T}}\left(t_{1}, \ldots, t_{n}\right), O W A_{W_{D}}\left(d_{1}, \ldots, d_{n}\right)\right)
$$

with following associated sets of normalised and positive trust weights $W_{T}=\left(w_{T_{1}}, \ldots w_{T_{n}}\right)$

$$
w_{T_{i}}=\frac{2 \cdot \max \left(0,\left[\frac{n}{2}-i+1\right]\right)}{\left[\frac{n}{2}\right]\left[\frac{n}{2}+1\right]}
$$

and normalised and positive distrust weights $W_{D}=\left(w_{D_{1}}, \ldots w_{D_{n}}\right)$ :

$$
w_{D_{i}}=\frac{2 \cdot \max \left(0,\left[\frac{n}{4}-i+1\right]\right)}{\left[\frac{n}{4}\right]\left[\frac{n}{4}+1\right]}
$$

The K-OWA aggregation operator has fixed orness $\left(W_{T}\right)=\operatorname{orness}\left(W_{D}\right)=2 / 3$, and then it always exhibits a maximum-like behaviour. However, in a real decision making case, this assumption may not be met because the group of experts may have different risk attitude such as: minimum-like, neutral-like behaviour, etc. Therefore, the K-OWA aggregation operator has a limitation of not allowing the implementation of different attitude of experts. To resolve this problem, the trust score induced ordered weighted operator (TS-IOWA) operator is proposed, which extends the induced ordered weighted averaging (IOWA) operator proposed by Yager [60] to the aggregation of orthopairs of trust/distrust values : 
Definition 6 (IOWA Operator). An IOWA operator is a mapping IOWA $_{W}:(\mathbb{R} \times \mathbb{R})^{n} \longrightarrow \mathbb{R}$, to which a set of positive and normalised vector is associated, $W=\left(w_{1}, \ldots, w_{n}\right)$, that aggregates the set of second arguments of a list of $n 2$-tuples $\left\{\left\langle u_{1}, p_{1}\right\rangle, \ldots,\left\langle u_{n}, p_{n}\right\rangle\right\}$ according to the following expression,

$$
\operatorname{IOWA}_{W}\left(\left\langle u_{1}, p_{1}\right\rangle, \ldots,\left\langle u_{n}, p_{n}\right\rangle\right)=\sum_{i=1}^{n} w_{i} \cdot p_{\sigma(i)},
$$

being $\sigma:\{1, \ldots, n\} \longrightarrow\{1, \ldots, n\}$ a permutation such that $u_{\sigma(i)} \geq u_{\sigma(i+1)}, \forall i=1, \ldots, n-1$, i.e., $\left\langle u_{\sigma(i)}, p_{\sigma(i)}\right\rangle$ is the 2 -tuple with $u_{\sigma(i)}$ the i-th highest value in the set $\left\{u_{1}, \ldots, u_{n}\right\}$.

Given a set of $n$ orthopairs of trust/distrust values $\left\{\left(t_{1}, d_{1}\right), \ldots,\left(t_{n}, d_{n}\right)\right\}$, their associated trust score and knowledge deficit vector $U=\left(u_{1}, \ldots, u_{n}\right)$ can be computed as per Definition 2

$$
u_{i}=\left(T S_{i}, K D_{i}\right)=\left(t_{i}-d_{i},\left|1-t_{i}-d_{i}\right|\right)
$$

and its components being ordered according to Definition 3, to be used as the order induce variable of the TS-IOWA operator. This is expressed formally in the following:

Definition 7 (TS-IOWA Operator). Given the set $\left\{\left(t_{i}, d_{i}\right) \mid i=1, \ldots, n\right\}$, the trust induced ordered weighted operator (TS-IOWA) with order induce variable $U=\left(u_{1}, \ldots, u_{n}\right), u_{i}=\left(T S_{i}, K D_{i}\right)=$ $\left(t_{i}-d_{i},\left|1-t_{i}-d_{i}\right|\right)$, is expressed as

$$
\left.\operatorname{TS}_{-I_{O W A}}\left(\left(t_{1}, d_{1}\right), \ldots,\left(t_{n}, d_{n}\right)\right)\right)=\left(\operatorname{IOWA}_{U, T}, \mathrm{IOWA}_{U, D}\right)
$$

where

$$
\operatorname{IOWA}_{U, T}=\operatorname{IOWA}\left(\left\langle u_{1}, t_{1}\right\rangle, \ldots,\left\langle u_{n}, t_{n}\right\rangle\right)
$$

and

$$
\operatorname{IOWA}_{U, D}=\operatorname{IOWA}\left(\left\langle u_{1}, d_{1}\right\rangle, \ldots,\left\langle u_{n}, d_{n}\right\rangle\right)
$$

An issue to deal with for the implementing the TS-IOWA operator is how to obtain its associated weighting vector. In our decision-making context, trust scores can also be used to assign importance degrees so that the higher the trust score the higher the importance degree $[11,43]$.

In [59], Yager provided a procedure to evaluate the overall satisfaction of $Q$ important criteria (experts) by an alternative $x$ by computing the weighting vector associated to an OWA operator as follows:

$$
w_{h}=Q\left(\frac{S(h)}{S(n)}\right)-Q\left(\frac{S(h-1)}{S(n)}\right)
$$

being $Q$ the Basic Unit-interval Monotone (BUM) membership function (non-decreasing $Q:[0,1] \rightarrow$ $[0,1]$ such that $Q(0)=0, Q(1)=1)$ of the linguistic quantifier, $S(h)=\sum_{l=1}^{h} s_{\sigma(l)}, s_{l}$ the importance degree of criterion $l$, and $\sigma$ the permutation used to produce the ordering of the values to be aggregated. 


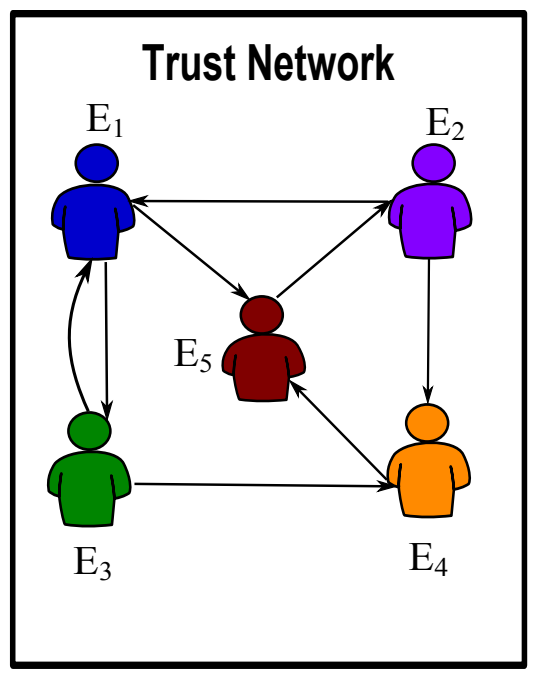

Figure 5: Graph representation of the trust sociomatrix

This approach for the inclusion of importance degrees associates a zero weight to those experts with zero importance degree. This procedure was extended to the case of IOWA operator in [60]. In this case, each component in the aggregation consists of a triple $\left(r_{h}, s_{h}, u_{h}\right)$ where $r_{h}$ is the argument value to aggregate, $s_{h}$ is the importance weight value associated to $r_{h}$, and $u_{h}$ is the order inducing value.

In this paper, each orthopair of trust/distrust values $\left(t_{h}, d_{h}\right)$ is associated its trust score $T S_{h}$ as its importance weight and its $u_{h}=\left(T S_{h}, K D_{h}\right)$ value as the order inducing value. Thus, the weights associated to the TS-IOWA operator in Definition 7 are computed as follows

$$
w_{\sigma(h)}=Q\left(\frac{T(\sigma(h))}{T(\sigma(k))}\right)-Q\left(\frac{T(\sigma(h-1))}{T(\sigma(k))}\right)
$$

with $T(\sigma(h))=\sum_{l=1}^{h} T S_{\sigma(l)}$, and $u_{\sigma(l)}$ is the $l$-th largest value of set $\left\{u_{1}, \ldots, u_{n}\right\}$.

It is worth noting that the TS-IOWA operator allows for different (BUM) functions $Q$ to be used, which allows for the implementation of different risk attitude of experts [59], Thus, the TS-IOWA operator is more flexible than the K-OWA operator as it is possible to be used in a wider range of decision contexts. In the following, we illustrate how the propagation operator and the TS-IOWA operator are used to complete a trust network sociomatrix.

Example 1. Suppose that five experts $\left\{E_{1}, E_{2}, E_{3}, E_{4}, E_{5}\right\}$ are socially trust related as illustrated in Figure 5, with following trust sociomatrix $S_{L}$ :

$$
S^{T}=\left(\begin{array}{ccccc}
- & - & (0.8,0.2) & - & (0.7,0.1) \\
(0.8,0.1) & - & - & (0.9,0.3) & - \\
(0.9,0.2) & - & - & (0.8,0.2) & - \\
- & - & - & - & (0.9,0.3) \\
- & (1.0,0.1) & - & - & -
\end{array}\right)
$$


Figure 5 is a connected network because there is a path between every pair of nodes but it is not complete because some pairs of nodes are not directly linked $[47,50]$. For example, there is no direct trust link from $E_{1}$ to $E_{4}$. However, we observe that there are two paths that goes from $E_{1}$ to $E_{4}$ : $E_{1} \rightarrow E_{3} \rightarrow E_{4}$ and $E_{1} \rightarrow E_{5} \rightarrow E_{2} \rightarrow E_{4}$. Then, according to expression (4), we can propagate trust and obtain the following orthopairs of trust/distrust between $E_{1}$ and $E_{4}$ :

$$
P_{134}((0.8,0.2),(0.8,0.2))=(0.64,0.16)
$$

and

$$
\left.P_{1524}((0.7,0.1),(1.0,0.1),(0.9,0.3))\right)=(0.63,0.21)
$$

The trust score and knowledge deficit of both paths are: $u_{134}=(0.48,0.2)$ and $u_{1524}=(0.42,0.16)$. To arrive to a single orthopair of trust/distrust value for the direct link from $E_{1}$ to $E_{4}$, we aggregate both $P_{134}$ and $P_{1524}$ using the TS-IOWA operator. The application of the quantifier $Q(r)=r^{0.5}$, used in literature to model the fuzzy linguistic quantifier 'most of' [11], yields the following weights

$$
w_{\sigma(1)}=0.73, \quad w_{\sigma(2)}=0.27
$$

and the final TS-IOWA orthopair of trust/distrust value from $E_{1}$ to $E_{4}$ of

$$
\left(t_{14}, d_{14}\right)=(0.73 \times 0.64+0.27 \times 0.63,0.73 \times 0.16+0.27 \times 0.21)=(0.64,0.17) .
$$

Applying the above same process, we arrive to the following complete trust sociomatrix

$$
S^{T}=\left(\begin{array}{ccccc}
- & (0.64,0.06) & (0.80,0.20) & (0.64,0.17) & (0.70,0.10) \\
(0.80,0.10) & - & (0.64,0.16) & (0.90,0.30) & (0.74,0.22) \\
(0.90,0.20) & (0.70,0.07) & - & (0.80,0.10) & (0.65,0.13) \\
(0.72,0.09) & (0.90,0.09) & (0.58,0.14) & - & (0.90,0.30) \\
(0.80,0.10) & (1.00,0.10) & (0.64,0.16) & (0.80,0.24) & -
\end{array}\right)
$$

\section{Trust Based Estimation and Aggregation of 2-tuple Linguistic Values}

To deal with the unknown elements in decision making process, some estimation methods were introduced in the decision making with preference relations $[1,12,13,44]$. These methods are usually driven by the notion of consistency, which is related to the personal knowledge and reasonability of individual expert. This article, though, proposes a novel estimation method for incomplete MCGDM problems in which one expert can use other experts' knowledge to estimate the unknown preference values in his/her personal decision matrix [2]. To do that, we first compute the relative trust degree (RTD) from one expert to the other experts, and then propose an approach to estimate the unknown elements by aggregating other experts' judgement. Furthermore, the average trust degree (ATD) is defined as a reliable source to compute experts' weights for aggregating the individual 2-tuple decision matrices. 


\subsection{Incomplete 2-tuple linguistic decision matrix}

Subjectivity, imprecision and vagueness in the articulation of opinions pervade real world decision applications, and individuals might feel more comfortable using words by means of linguistic labels or terms to articulate their preferences $[36,39,62]$. In these cases is still valid the following quotation by Zadeh [63]: 'Since words, in general, are less precise than numbers, the concept of a linguistic variable serves the purpose of providing a means of approximate characterisation of phenomena which are too complex or too ill-defined to be amenable to description in conventional quantitative terms.'

Let $\mathcal{S}=\left\{s_{0}, \ldots, s_{t}\right\}$ be a set of linguistic labels $(t \geq 2)$, with semantic underlying a ranking relation that can be precisely captured with a linear order, i.e., $s_{0}<s_{1}<\cdots<s_{t}$. Table 2 provides an example with seven linguistic labels and their corresponding semantic meanings.

\begin{tabular}{|c|l|}
\hline Linguistic label & Semantic meaning \\
\hline$s_{0}$ & extremely poor (EP) \\
$s_{1}$ & very poor (VP) \\
$s_{2}$ & poor (P) \\
$s_{3}$ & medium/average (M) \\
$s_{4}$ & good (G) \\
$s_{5}$ & very good (VG) \\
$s_{6}$ & extremely good (EG) \\
\hline
\end{tabular}

Table 2: Seven linguistic labels and their semantic meanings

Assuming that the number of labels is odd and the central label $s_{t / 2}$ stands for the medium/average state, the remaining labels are usually located symmetrically around that central assessment. Thus, the operator defined as $N\left(s_{h}\right)=s_{g}$ with $(g+h)=t$ is a negator operator because $N\left(N\left(s_{h}\right)\right)=N\left(s_{g}\right)=s_{h}$ [40].

The 2-tuple linguistic model was introduced by Herrera and Martínez in [26]. This linguistic model takes as a basis the symbolic representation model based on indexes and in addition defines the concept of symbolic translation to represent the linguistic information by means of a pair of values called linguistic 2-tuple, $\left(s_{b}, \alpha_{b}\right)$, where $s_{b} \in \mathcal{S}$ is one of the original linguistic terms and $\alpha_{b}$ is a numeric value representing the symbolic translation. This representation structure allows, on the one hand, to obtain the same information than with the symbolic representation model based on indexes without losing information in the aggregation phase. On the other hand, the result of the aggregation is expressed on the same domain as the one of the initial linguistic labels and therefore, the well-known re-translation problem of the above methods is avoided.

Definition 8 (Linguistic 2-tuple representation). Let $a \in[0, t]$ be the result of a symbolic aggregation of the indexes of a set of labels assessed in a linguistic term set $\mathcal{S}=\left\{s_{0}, \ldots, s_{t}\right\}$. Let 
$b=\operatorname{round}(a) \in\{0, \ldots, t\}$. The value $\alpha_{b}=a-b \in[-0.5,0.5)$ is called a symbolic translation, and the pair of values $\left(s_{b}, \alpha_{b}\right)$ is called the 2 -tuple linguistic representation of the symbolic aggregation $a$.

The 2-tuple linguistic representation of symbolic aggregation can be mathematically formalised with the following mapping:

$$
\begin{gathered}
\Delta:[0, t] \longrightarrow \mathcal{S} \times[-0.5,0.5) \\
\Delta(a)=\left(s_{b}, \alpha_{b}\right) .
\end{gathered}
$$

Based on the linear order of the linguistic term set and the complete ordering of the set $[-0.5,0.5)$, it is easy to prove that $\Delta$ is strictly increasing and continuous and, therefore its inverse function exists:

$$
\begin{gathered}
\Delta^{-1}: \mathcal{S} \times[-0.5,0.5) \longrightarrow[0, t] \\
\Delta^{-1}\left(s_{b}, \alpha_{b}\right)=b+\alpha_{b}=a .
\end{gathered}
$$

The following negation operator is defined: $N(\Delta(a))=\Delta(t-a)$. Figure 6 illustrates the application of the 2-tuple function $\Delta$ and its inverse $\Delta^{-1}$ for a linguistic term set of cardinality seven. The value of the symbolic translation is assumed to be 2.8 , which means that $\operatorname{round}(2.8)=3$ and therefore it can be represented with the $2-$ tuple $\left(s_{3},-0.2\right)$.

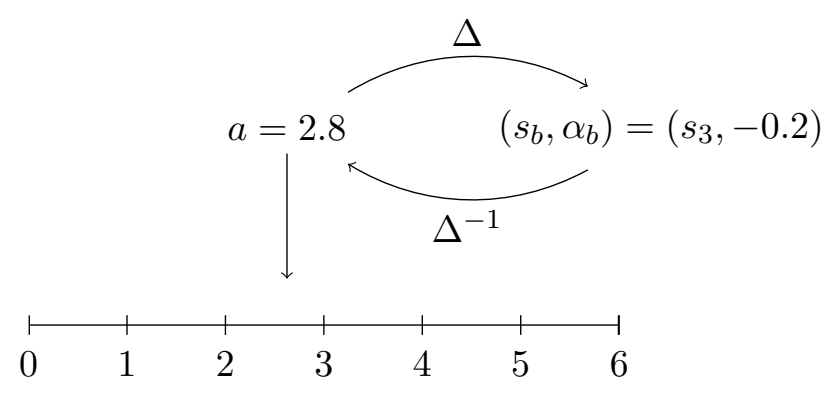

Figure 6: Linguistic 2-tuple symbolic translation

Give a set of 2-tuple linguistic labels $\left\{\left(s_{1}, \alpha_{1}\right),\left(s_{2}, \alpha_{2}\right), \ldots,\left(s_{n}, \alpha_{n}\right)\right\}$ and a set of positive and normalised weights $\left(w_{1}, w_{2}, \ldots, w_{n}\right)^{T}$, i.e. $w_{j} \in[0,1], \sum_{j=1}^{n} w_{j}=1$, the 2 -tuples weighted arithmetic average (WAA) is computed as follows [3]:

$$
(\widetilde{s}, \widetilde{\alpha})=\varphi\left(\left(s_{1}, \alpha_{1}\right), \ldots,\left(s_{n}, \alpha_{n}\right)\right)=\Delta\left(\sum_{j=1}^{n} w_{j} \cdot \Delta^{-1}\left(s_{i}, \alpha_{i}\right)\right)
$$

where $\widetilde{s} \in \mathcal{S}, \widetilde{\alpha} \in[-0.5,0.5)$.

Definition 9 (2-tuple decision matrix). A 2-tuple decision matrix decision matrix $R=\left(r_{i j}\right)_{m \times n}$ is a matrix with elements $r_{i j}$ being 2 -tuple linguistic labels:

$$
r_{i j}=\left(s_{i j}, \alpha_{i j}\right), i=1,2, \ldots, m ; j=1,2, \ldots, n
$$

If an expert is not able to efficiently provide the preference values to some of the alternatives under a particular criterion, then an incomplete 2-tuple decision matrix will result [1]. The 2-tuple 
decision matrix can be considered as a particular case of the incomplete 2-tuple decision matrix. For an incomplete 2-tuple decision matrices, when the preference value of alternatives $x_{i}$ under criteria $c_{j}, r_{i j}$, is not known the symbol $\times$ will be used in the corresponding matrix entry.

Example 2. A company is to evaluate four green suppliers: $\left\{x_{1}, x_{2}, x_{3}, x_{4}\right\}$. The following five criteria $\left\{c_{1}, c_{2}, c_{3}, c_{4}, c_{5}\right\}$ are considered: $c_{1}=$ Remanufacturing/reuse activity; $c_{2}=$ Energy consumption; $c_{3}=$ Reverse logistics program; $c_{4}=$ Hazardous waste management; $c_{5}=$ Environmental certification. The weights associated to the five criteria is $\omega=(0.25,0.20,0.15,0.10,0.30)^{T}$. This company has a group of experts $\left\{E_{1}, E_{2}, E_{3}, E_{4}, E_{5}\right\}$ form five different departments. The five experts give the following linguistics evaluations of four green suppliers:

\begin{tabular}{c|ccccc} 
& \multicolumn{5}{|c}{ Expert $E_{1}$ assessments } \\
\hline Supplier/Criterion & $C_{1}$ & $C_{2}$ & $C_{3}$ & $C_{4}$ & $C_{5}$ \\
\hline$A_{1}$ & $\mathrm{G}$ & $\mathrm{VG}$ & $\mathrm{G}$ & $\mathrm{P}$ & $\mathrm{M}$ \\
$A_{2}$ & $\times$ & $\mathrm{M}$ & $\mathrm{VG}$ & $\mathrm{M}$ & $\times$ \\
$A_{3}$ & $\mathrm{VG}$ & $\mathrm{P}$ & $\times$ & $\mathrm{VP}$ & $\mathrm{G}$ \\
$A_{4}$ & $\mathrm{G}$ & $\mathrm{G}$ & $\mathrm{VG}$ & $\mathrm{M}$ & $\mathrm{VG}$ \\
\hline
\end{tabular}

Expert $E_{2}$ assessments
\begin{tabular}{|ccccc|}
\hline$C_{1}$ & $C_{2}$ & $C_{3}$ & $C_{4}$ & $C_{5}$ \\
\hline $\mathrm{P}$ & $\mathrm{G}$ & $\times$ & $\mathrm{M}$ & $\mathrm{M}$ \\
$\mathrm{EG}$ & $\mathrm{VG}$ & $\mathrm{M}$ & $\mathrm{G}$ & $\mathrm{P}$ \\
$\times$ & $\mathrm{M}$ & $\mathrm{G}$ & $\mathrm{M}$ & $\mathrm{M}$ \\
$\mathrm{VG}$ & $\mathrm{G}$ & $\mathrm{VG}$ & $\mathrm{P}$ & $\mathrm{G}$ \\
\hline
\end{tabular}

\begin{tabular}{|ccccc|}
\hline$C_{1}$ & $C_{2}$ & $C_{3}$ & $C_{4}$ & $C_{5}$ \\
\hline $\mathrm{M}$ & $\mathrm{G}$ & $\mathrm{G}$ & $\mathrm{P}$ & $\mathrm{G}$ \\
$\mathrm{G}$ & $\mathrm{M}$ & $\times$ & $\mathrm{M}$ & $\mathrm{M}$ \\
$\mathrm{VG}$ & $\mathrm{M}$ & $\mathrm{M}$ & $\mathrm{P}$ & $\mathrm{VG}$ \\
$\mathrm{VG}$ & $\mathrm{P}$ & $\mathrm{M}$ & $\mathrm{VP}$ & $\mathrm{M}$ \\
\hline
\end{tabular}

\begin{tabular}{c|ccccc} 
& \multicolumn{5}{|c}{ Expert $E_{4}$ assessments } \\
\hline Supplier/Criterion & $C_{1}$ & $C_{2}$ & $C_{3}$ & $C_{4}$ & $C_{5}$ \\
\hline$A_{1}$ & $\mathrm{G}$ & $\times$ & $\mathrm{VG}$ & $\mathrm{M}$ & $\mathrm{P}$ \\
$A_{2}$ & $\mathrm{VG}$ & $\mathrm{G}$ & $\mathrm{G}$ & $\mathrm{P}$ & $\mathrm{G}$ \\
$A_{3}$ & $\mathrm{G}$ & $\mathrm{VP}$ & $\mathrm{M}$ & $\mathrm{M}$ & $\mathrm{P}$ \\
$A_{4}$ & $\mathrm{EG}$ & $\mathrm{M}$ & $\times$ & $\mathrm{M}$ & $\mathrm{VG}$ \\
\hline
\end{tabular}

\begin{tabular}{|ccccc|}
\multicolumn{7}{c}{ Expert $E_{5}$ assessments } \\
\hline$C_{1}$ & $C_{2}$ & $C_{3}$ & $C_{4}$ & $C_{5}$ \\
\hline $\mathrm{M}$ & $\mathrm{VG}$ & $\mathrm{VG}$ & $\times$ & $\times$ \\
$\mathrm{VG}$ & $\mathrm{G}$ & $\mathrm{M}$ & $\mathrm{G}$ & $\mathrm{G}$ \\
$\mathrm{EG}$ & $\mathrm{P}$ & $\mathrm{P}$ & $\mathrm{M}$ & $\mathrm{M}$ \\
$\mathrm{G}$ & $\mathrm{M}$ & $\mathrm{G}$ & $\mathrm{P}$ & $\mathrm{VG}$ \\
\hline
\end{tabular}

The incomplete 2 -tuple decision matrices representation are:

$$
\begin{aligned}
R^{1} & =\left(\begin{array}{ccccc}
\left(s_{4}, 0\right) & \left(s_{5}, 0\right) & \left(s_{4}, 0\right) & \left(s_{2}, 0\right) & \left(s_{3}, 0\right) \\
\times & \left(s_{3}, 0\right) & \left(s_{5}, 0\right) & \left(s_{3}, 0\right) & \times \\
\left(s_{5}, 0\right) & \left(s_{2}, 0\right) & \times & \left(s_{1}, 0\right) & \left(s_{4}, 0\right) \\
\left(s_{4}, 0\right) & \left(s_{4}, 0\right) & \left(s_{5}, 0\right) & \left(s_{3}, 0\right) & \left(s_{5}, 0\right)
\end{array}\right) \quad R^{2}=\left(\begin{array}{cccccc}
\left(s_{2}, 0\right) & \left(s_{4}, 0\right) & \times & \left(s_{3}, 0\right) & \left(s_{3}, 0\right) \\
\left(s_{6}, 0\right) & \left(s_{5}, 0\right) & \left(s_{3}, 0\right) & \left(s_{4}, 0\right) & \left(s_{2}, 0\right) \\
\times & \left(s_{3}, 0\right) & \left(s_{4}, 0\right) & \left(s_{3}, 0\right) & \left(s_{3}, 0\right) \\
\left(s_{5}, 0\right) & \left(s_{4}, 0\right) & \left(s_{5}, 0\right) & \left(s_{2}, 0\right) & \left(s_{4}, 0\right)
\end{array}\right) \\
R^{3} & =\left(\begin{array}{cccccc}
\left(s_{3}, 0\right) & \left(s_{4}, 0\right) & \left(s_{4}, 0\right) & \left(s_{2}, 0\right) & \left(s_{4}, 0\right) \\
\left(s_{4}, 0\right) & \left(s_{3}, 0\right) & \times & \left(s_{3}, 0\right) & \left(s_{3}, 0\right) \\
\left(s_{5}, 0\right) & \left(s_{3}, 0\right) & \left(s_{3}, 0\right) & \left(s_{2}, 0\right) & \left(s_{5}, 0\right) \\
\left(s_{5}, 0\right) & \left(s_{2}, 0\right) & \left(s_{3}, 0\right) & \left(s_{1}, 0\right) & \left(s_{3}, 0\right)
\end{array}\right) \quad R^{4}=\left(\begin{array}{ccccc}
\left(s_{4}, 0\right) & \times & \left(s_{5}, 0\right) & \left(s_{3}, 0\right) & \left(s_{2}, 0\right) \\
\left(s_{5}, 0\right) & \left(s_{4}, 0\right) & \left(s_{4}, 0\right) & \left(s_{2}, 0\right) & \left(s_{4}, 0\right) \\
\left(s_{4}, 0\right) & \left(s_{1}, 0\right) & \left(s_{3}, 0\right) & \left(s_{3}, 0\right) & \left(s_{2}, 0\right) \\
\left(s_{6}, 0\right) & \left(s_{3}, 0\right) & \times & \left(s_{3}, 0\right) & \left(s_{5}, 0\right)
\end{array}\right) \\
& \\
R^{5} & =\left(\begin{array}{lllll}
\left(s_{3}, 0\right) & \left(s_{5}, 0\right) & \left(s_{5}, 0\right) & \times & \times \\
\left(s_{5}, 0\right) & \left(s_{4}, 0\right) & \left(s_{3}, 0\right) & \left(s_{4}, 0\right) & \left(s_{4}, 0\right) \\
\left(s_{6}, 0\right) & \left(s_{2}, 0\right) & \left(s_{2}, 0\right) & \left(s_{3}, 0\right) & \left(s_{3}, 0\right) \\
\left(s_{4}, 0\right) & \left(s_{3}, 0\right) & \left(s_{4}, 0\right) & \left(s_{2}, 0\right) & \left(s_{5}, 0\right)
\end{array}\right)
\end{aligned}
$$

\subsection{Trust based estimation of unknown 2-tuple linguistic values}

Recall that we are assuming a group of experts, $E=\left\{E_{1}, \ldots, E_{k}\right\}$, are related via a connected trust network as illustrated in Fig. 5. The trust propagation operator in conjunction with the TS-IOWA 
operator are used to make this trust network a complete one and consequently all network ordered pairs of nodes, $\left(E_{l}, E_{h}\right)$, will have a directed link with an associated orthopairs of trust/distrust from $E_{l}$ to $E_{h}: S_{l h}=\left(t_{l h}, d_{l h}\right)$. Note that each expert, $E_{l}$, can be associated its trust scores to each one of the other experts in the network: $\left\{T S_{l f}=t_{l f}-d_{l f} \mid f \in\{1, \ldots, k\} \wedge f \neq l\right\}$.

The problem here is how to arrive at consensus between this group of experts regarding the selection of the best alternative from a set of feasible ones, $A=\left\{x_{1}, \ldots, x_{m}\right\}$, that are assessed linguistically using a set of labels, $\mathcal{S}=\left\{s_{0}, \ldots, s_{t}\right\}$, against a set of criteria, $\left\{C_{1}, \ldots, C_{n}\right\}$. Thus, each expert, $E_{l}$, will be providing a linguistic decision matrix that will be conveniently represented in the form of a 2-tuple linguistic decision matrix, $R^{l}=\left(r_{i j}^{l}\right)_{m \times n}$.

We note that trust score values of a particular expert can be used to compute a trust based estimated decision matrix using the decision matrices of the other experts in the group, which can be useful if a particular expert provides an incomplete decision matrix. Indeed, we first remark that the following set of relative trust score values

$$
\left\{R T S_{l h}=\frac{T S_{l h}}{\sum_{\substack{f=1 \\ f \neq l}}^{k} T S_{l f}} \mid h \in\{1, \ldots, k\} \wedge h \neq l\right\}
$$

constitutes a normalised weighting vector, and therefore can be used as weighting vector of the 2 -tuples weighted arithmetic average (WAA) to aggregate the rest of experts decision matrices

$$
\widetilde{r}_{i j}^{l}=\Delta\left(\sum_{\substack{h=1 \\ h \neq l}}^{k} R T S_{l h} \cdot \Delta^{-1}\left(r_{i j}^{h}\right)\right)
$$

This aggregated value is derived using the knowledge of the rest of experts, and will be referred here simply as the trust based estimated assessment, and can be used to estimate a particular linguistic assessment when not provided by just one expert, as the following example illustrates:

Example 3. (Example 2 continuation) Using the complete trust sociomatrix $S_{T}$ obtained in Example 1, we compute the relative trust score (RTD) matrix:

$$
R T S=\left(\begin{array}{ccccc}
- & 0.25 & 0.27 & 0.21 & 0.27 \\
0.30 & - & 0.21 & 0.26 & 0.23 \\
0.27 & 0.25 & - & 0.27 & 0.21 \\
0.25 & 0.33 & 0.18 & 0 & 0.24 \\
0.27 & 0.34 & 0.18 & 0.21 & 0
\end{array}\right)
$$

Applying expression (17), the unknown preference values in $R^{1}, R^{2}, R^{3}, R^{4}, R^{5}$ are estimated with the following trust based 2-tuple values:

$\widetilde{r}_{21}^{1}=\left(s_{5}, 0\right), \widetilde{r}_{25}^{1}=\left(s_{3}, 0.2\right), \widetilde{r}_{33}^{1}=\left(s_{3}, 0\right), \widetilde{r}_{13}^{2}=\left(s_{4}, 0.5\right), \widetilde{r}_{31}^{2}=\left(s_{5}, 0\right), \widetilde{r}_{23}^{3}=\left(s_{4},-0.2\right), \widetilde{r}_{12}^{4}=\left(s_{4}, 0.5\right)$, $\widetilde{r}_{43}^{4}=\left(s_{5},-0.4\right), \widetilde{r}_{14}^{5}=\left(s_{3},-0.4\right), \widetilde{r}_{15}^{5}=\left(s_{3}, 0\right)$ 
The above estimation procedure can be extended to the case of having more than one expert not providing the same linguistic evaluation and there is at least one expert providing it. Indeed, denoting $M V_{i j}=\left\{f \in\{1, \ldots, k\} \mid r_{i j}^{f}\right.$ is unknown $\}$ then we adapt expression (17) as follows:

$$
\forall l \in M V_{i j}: \widetilde{r}_{i j}^{l}=\Delta\left(\sum_{h \notin M V_{i j}} \overline{R T S}_{l h} \cdot \Delta^{-1}\left(r_{i j}^{h}\right)\right)
$$

with $\overline{R T S}_{l h}=\frac{T S_{l h}}{\sum_{f \notin M V_{i j}} T S_{l f}}$.

\subsection{Trust aggregation of 2-tuple linguistic decision matrices}

From previous section, we can assume that experts decision matrices are complete, and thus they are in a position to aggregate them in order to choose a collective decision. As it happens that the experts are trust networked, the aggregation step can be used to implement the different degrees of trust an expert receives from the rest of the group as a measure of its prominent position within the network. In SNA, for a weighted directed network like the trust network here dealt with, the in-degree centrality is used to quantify the importance of the nodes in the network [47,50]. Using averages, the trust score in-degree centrality associated to expert $E_{l}$ is

$$
T S_{h}=\frac{1}{k-1} \sum_{l=1}^{k} T S_{l h}
$$

Thus, each expert in the trust network can be associated the following normalised average trust degree (ATD)

$$
A T D_{h}=\frac{T S_{h}}{\sum_{l=1}^{k} T S_{l}}
$$

This value will be used as the contribution of an expert in the collective 2 -tuple linguistic decision matrix, $R=\left(\bar{r}_{i j}\right)$ :

$$
\bar{r}_{i j}=\Delta\left(\sum_{h=1}^{k} A T D_{h} \cdot \Delta^{-1}\left(r_{i j}^{h}\right)\right) .
$$

Example 4. (Example 2 continuation) According to expression (19), the trust score in-degree centrality values of experts $E_{1}, E_{2}, E_{3}, E_{4}, E_{5}$ are:

$$
T S_{1}=0.69 T S_{2}=0.73 ; T S_{3}=0.5 ; T S_{4}=0.59 ; \quad T S_{5}=0.56 .
$$

Thus, the average trust degrees of each expert are:

$$
A T D_{1}=0.23 ; A T D_{2}=0.24 ; A T D_{3}=0.16 ; A T D_{4}=0.19 ; A T D_{5}=0.18 .
$$


The collective 2 -tuple linguistic decision matrix is

$$
\bar{R}=\left(\begin{array}{ccccc}
\left(s_{3}, 0.2\right) & \left(s_{4}, 0.5\right) & \left(s_{4}, 0.5\right) & \left(s_{2}, 0.5\right) & \left(s_{3}, 0\right) \\
\left(s_{5}, 0.1\right) & \left(s_{4},-0.1\right) & \left(s_{4},-0.2\right) & \left(s_{3}, 0.2\right) & \left(s_{3}, 0.2\right) \\
\left(s_{5}, 0\right) & \left(s_{2}, 0.2\right) & \left(s_{3}, 0.1\right) & \left(s_{2}, 0.4\right) & \left(s_{3}, 0.4\right) \\
\left(s_{5},-0.2\right) & \left(s_{3}, 0.3\right) & \left(s_{4}, 0.4\right) & \left(s_{2}, 0.3\right) & \left(s_{4}, 0.4\right)
\end{array}\right)
$$

\section{Visual consensus model for incomplete MCGDM}

Once the collective 2-tuple decision matrix is obtained, we define the consensus index (CI) at three levels: (i) decision matrix; (ii) alternatives; and (iii) preference values. When the consensus index reaches a threshold value, agreed by the group of experts, the resolution process of the MCGDM is carried out; otherwise the experts are normally invited to revisit their opinions further in an effort to make them closer. To do that, a visual consensus model is here developed to help experts 'see' their relative consensus position within the group as well as to identify the alternatives and preference values that contribute less to consensus. Additionally, the visual consensus model also includes a recommendation mechanism to produce individualised advise to experts on how to increase consensus. Finally, a visual graphical simulation of future consensus status if the recommended values were to be implemented is provided. In the light of this visual extra information, experts can revisit their evaluations and make changes if considered appropriate to increase consensus.

The trust propagating aggregation and visual consensus decision model for incomplete MCGDM with 2-tuple linguistic information is depicted in Figure 7. Specifically, it consists of the following five steps: (1) Computing trust degrees; (2) Estimating unknown preference values; (3) Determining the consensus index at the three levels; (4) Visual consensus identification, recommendation and feedback simulation; and (5) Selection Process. The first and second steps have already been covered in Section 2 and Section 3, respectively. The remaining steps will be presented in more detail in the following subsections. A step-by-step example to illustrate the computation processes involved in each step is also provided. For the sake of simplicity, a low number of experts and alternatives are assumed.

\subsection{Consensus Index}

Key in the visual consensus model is the computation of the consensus index for each expert measuring the similarity between an expert's provided preferences and the group of experts' collective preferences [14, 42]:

Level 1. Consensus index on preference values. The consensus index of an expert $E_{h}$ to the group of expert on the assessment of alternative $x_{i}$ under criterion $C_{j}$ is

$$
C E_{i j}^{h}=1-d\left(r_{i j}^{h}, \bar{r}_{i j}\right)=1-\frac{\left|\Delta^{-1}\left(r_{i j}^{h}\right)-\Delta^{-1}\left(\bar{r}_{i j}\right)\right|}{t} .
$$




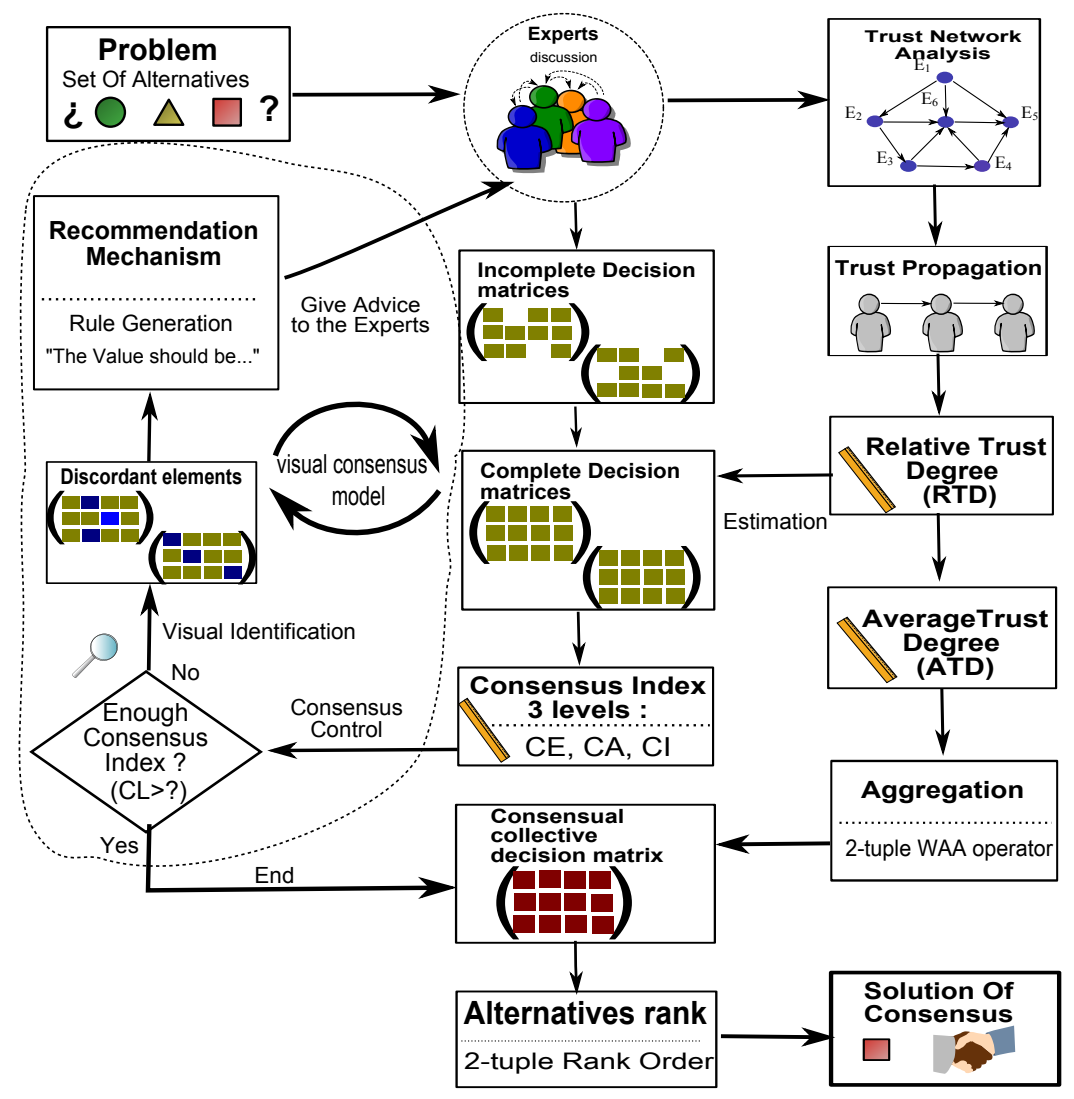

Figure 7: Trust propagating aggregation and visual consensus model for MCGDM under incomplete information

Level 2. Consensus index on alternatives. The consensus index of an expert $E_{h}$ to the group of expert on the alternative $x_{i}$ is

$$
C A_{i}^{h}=\frac{1}{n} \sum_{j=1}^{n} C E_{i j}^{h} .
$$

Level 3. Consensus index on the decision matrix. The consensus index of an expert $E_{h}$ to the group of expert on the decision matrix is

$$
C I^{h}=\frac{1}{m} \sum_{i=1}^{m} C A_{i}^{h} .
$$

The greater the value of $C I^{h}\left(0 \leq C I^{h} \leq 1\right)$, the greater the agreement between an individual expert $\left(E_{h}\right)$ and the group as a collective. When $\min _{h} C I^{h}$ is greater than a threshold value $\gamma \in[0.5,1)$, fixed a priori by the group of expert, then the consensus reaching process ends and the selection process is applied to achieve the solution of consensus. Otherwise, a recommendation mechanism could be activated to give advice to the experts.

Example 5. (Example 2 continuation) The consensus indexes on preference values of alternatives are:

$$
C E^{1}=\left(\begin{array}{ccccc}
0.87 & 0.92 & 0.92 & 0.92 & 1.00 \\
0.98 & 0.85 & 0.80 & 0.97 & 1.00 \\
1.00 & 0.97 & 0.98 & 0.77 & 0.90 \\
0.87 & 0.88 & 0.90 & 0.88 & 0.90
\end{array}\right) \quad C E^{2}=\left(\begin{array}{ccccc}
0.80 & 0.92 & 1.00 & 0.92 & 1.00 \\
0.85 & 0.82 & 0.87 & 0.87 & 0.80 \\
1.00 & 0.87 & 0.85 & 0.90 & 0.93 \\
0.97 & 0.88 & 0.90 & 0.95 & 0.93
\end{array}\right)
$$




$$
\begin{aligned}
C E^{3}= & \left(\begin{array}{lllll}
0.97 & 0.92 & 0.92 & 0.92 & 0.83 \\
0.82 & 0.85 & 1.00 & 0.97 & 0.97 \\
1.00 & 0.87 & 0.98 & 0.93 & 0.73 \\
0.97 & 0.78 & 0.77 & 0.78 & 0.77
\end{array}\right) \quad C E^{4}=\left(\begin{array}{llllll}
0.87 & 1.00 & 0.92 & 0.92 & 0.83 \\
0.98 & 0.98 & 0.97 & 0.80 & 0.87 \\
0.83 & 0.80 & 0.98 & 0.90 & 0.77 \\
0.80 & 0.95 & 0.97 & 0.88 & 0.90
\end{array}\right) \\
C E^{5} & =\left(\begin{array}{lllll}
0.97 & 0.92 & 0.92 & 0.98 & 1.00 \\
0.98 & 0.98 & 0.86 & 0.87 & 0.87 \\
0.83 & 0.97 & 0.82 & 0.90 & 0.93 \\
0.87 & 0.95 & 0.93 & 0.95 & 0.90
\end{array}\right)
\end{aligned}
$$

The consensus indexes on alternatives are:

$$
\begin{array}{ll}
C A^{1}=(0.92,0.92,0.92,0.89) ; & C A^{2}=(0.93,0.84,0.91,0.93) ; \quad C A^{3}=(0.91,0.92,0.90,0.81) ; \\
C A^{4}=(0.91,0.92,0.86,0.90) ; & C A^{5}=(0.96,0.91,0.89,0.92) .
\end{array}
$$

The individual consensus indexes are:

$$
C I^{1}=0.91, C I^{2}=0.90, C I^{3}=0.88, C I^{4}=0.89, C I^{5}=0.92 \text {. }
$$

With a threshold value of $\gamma=0.9$ the recommendation mechanism is activated to assist experts $E_{3}$ and $E_{4}$.

\subsubsection{Visual Identification of Preference Relation}

Visual graphs as illustrated in Figure 8 can help experts 'see' their consensus position within the group. Following with Example 5, Figure 8(a) presents a visual representation of all experts consensus levels with respect to the threshold value, which clearly identifies $E_{3}$ and $E_{4}$ as the two experts contributing less to group consensus. Additionally, individual visual representations of consensus levels of alternatives and preference values are also provided to each expert to help them identify those alternatives and their associated preference values that contribute less to consensus. For example, visual representation at these two levels for expert $E_{4}$ are illustrated in Figure 8(b) and Figure 8(c), respectively. Mathematically, these steps are modelled as follows:

Step 1. The experts with a consensus index lower than the threshold value $\gamma$ are identified:

$$
E X P C H=\left\{h \mid C I^{h}<\gamma\right\}
$$

Step 2. For the identified experts, their alternatives with a $C A_{i}^{h}$ lower than the satisfaction threshold $\gamma$ are identified:

$$
A L T=\left\{(h, i) \mid E_{h} \in E X P C H \wedge C A_{i}^{h}<\gamma\right\}
$$

Step 3. Finally, the preference values to be changed are:

$$
A P S=\left\{(h, i, j) \mid(h, i) \in A L T \wedge C E_{i j}^{h}<\gamma\right\} .
$$




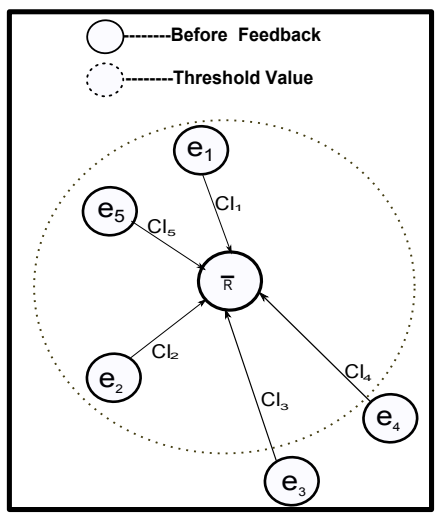

(a) Experts consensus index on the decision matrix

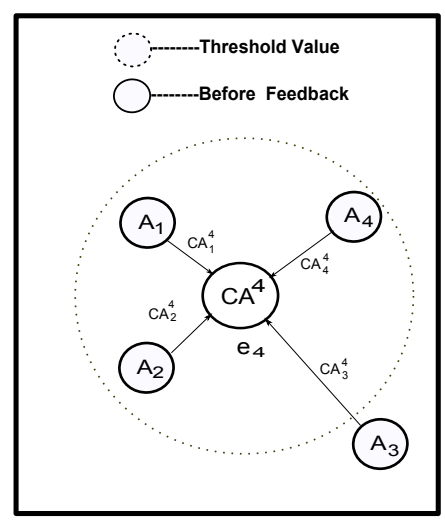

(b) Expert $E_{4}$ consensus index on all alternatives

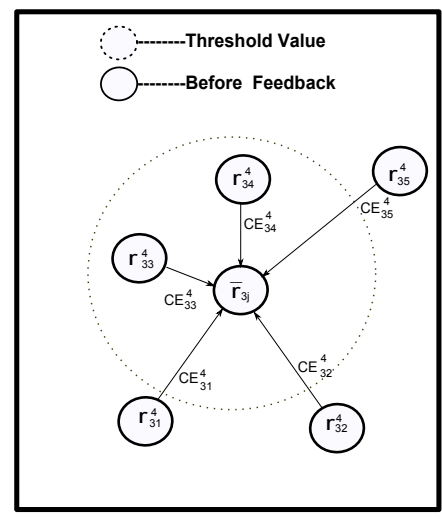

(c) Expert $E_{4}$ consensus index on preferences for alternative $A_{3}$

Figure 8: Visual representation of the three different levels of consensus

Example 6. (Example 3 continuation) The following APS set is obtained:

$$
A P S=\{(3,4,2),(3,4,3),(3,4,4),(3,4,5),(4,3,1),(4,3,2),(4,3,5)\}
$$

Then the generation of advice is activated.

\subsubsection{Recommendation Mechanism for Generating Advice}

The recommendation mechanism produces personalised advice to experts on which preference values they should consider to change as well as the new preference values to use in order to increase their consensus level. For all $(h, i, j) \in A P S$, the following personalised recommendation rule is presented to the corresponding expert:

"Your assessment of alternative $x_{i}$ under criterion $C_{j}, r_{i j}^{h}$, should change to a value closer to $r r_{i j}^{h}$."

$$
\Delta^{-1}\left(r r_{i j}^{h}\right)=\delta \cdot \Delta^{-1}\left(r_{i j}^{h}\right)+(1-\delta) \cdot \Delta^{-1}\left(\bar{r}_{i j}\right)
$$

where $\delta \in[0,1]$ is a parameter to control the percentage of change in value from the original assessment to the collective assessment. For $\delta=1$ no change is recommended, while for $\delta=0$ the original value is recommended to be completely replaced by the collective one.

Example 7. (Example 2 continuation) Taking a value of $\delta=0.5$, the recommendations for expert $E_{3}$ are:

- Your assessment of alternative $x_{4}$ under criteria $C_{2}$ should change to a value closer to $\left(s_{3},-0.3\right)$.

- Your assessment of alternative $x_{4}$ under criteria $C_{3}$ should change to a value closer to $\left(s_{4},-0.3\right)$.

- Your assessment of alternative $x_{4}$ under criteria $C_{4}$ should change to a value closer to $\left(s_{2},-0.3\right)$.

- Your assessment of alternative $x_{4}$ under criteria $C_{5}$ should change to a value closer to $\left(s_{4},-0.3\right)$. 
The recommendations for expert $E_{4}$ are:

- Your assessment of alternative $x_{3}$ under criteria $C_{1}$ should change to a value closer to $\left(s_{4}, 0.5\right)$.

- Your assessment of alternative $x_{3}$ under criteria $C_{2}$ should change to a value closer to $\left(s_{2},-0.4\right)$.

- Your assessment of alternative $x_{3}$ under criteria $C_{5}$ should change to a value closer to $\left(s_{3},-0.3\right)$.

\subsubsection{Visual Feedback Process Simulation}

The above recommendation mechanism has the advantage that the new preference values will be closer to the collective ones. As a consequence, when all experts' preferences are replaced by the corresponding recommended preference values, as per the feedback rule above, will guarantee that all expert consensus index values increase. This is proved in the following:

Lemma 1. Let $\left\{R^{h}=\left(r_{i j}^{h}\right) \mid h=1, \cdots, k\right\}$ be a set of 2-tuple linguistic decision matrices and $\bar{R}=\left(\bar{r}_{i j}\right)$ be the collective 2-tuple linguistic decision matrix

$$
\bar{r}_{i j}=\Delta\left(\sum_{h=1}^{k} A T D_{h} \cdot \Delta^{-1}\left(r_{i j}^{h}\right)\right) .
$$

Let $\left\{r R^{h}=\left(r r_{i j}^{h}\right) \mid h=1, \cdots, k\right\}$ be the set of 2-tuple linguistic decision matrices with

$$
\Delta^{-1}\left(r r_{i j}^{h}\right)=\delta \cdot \Delta^{-1}\left(r_{i j}^{h}\right)+(1-\delta) \cdot \Delta^{-1}\left(\bar{r}_{i j}\right)
$$

and $\overline{\bar{R}}=\left(\overline{\bar{r}}_{i j}\right)$ be the collective 2-tuple linguistic decision matrix

$$
\overline{\bar{r}}_{i j}=\Delta\left(\sum_{h=1}^{k} A T D_{h} \cdot \Delta^{-1}\left(r r_{i j}^{h}\right)\right) .
$$

We have:

$$
\overline{\bar{R}}=\bar{R}
$$

Proof. Indeed, we have:

$$
\begin{aligned}
\overline{\bar{r}}_{i j} & =\Delta\left(\sum_{h=1}^{k} A T D_{h} \cdot \Delta^{-1}\left(r r_{i j}^{h}\right)\right)=\Delta\left(\sum_{h=1}^{k} A T D_{h} \cdot\left(\delta \cdot \Delta^{-1}\left(r_{i j}^{h}\right)+(1-\delta) \cdot \Delta^{-1}\left(\bar{r}_{i j}\right)\right)\right) \\
& =\Delta\left(\delta \cdot \sum_{h=1}^{k} A T D_{h} \cdot \Delta^{-1}\left(r_{i j}^{h}\right)+(1-\delta) \cdot \Delta^{-1}\left(\bar{r}_{i j}\right) \cdot \sum_{h=1}^{k} A T D_{h}\right)=\Delta\left(\delta \cdot \Delta^{-1}\left(\bar{r}_{i j}\right)+(1-\delta) \cdot \Delta^{-1}\left(\bar{r}_{i j}\right)\right) \\
& =\bar{r}_{i j}
\end{aligned}
$$

Proposition 1. Under the condition of Lemma 1 we have: $d\left(r r_{i j}^{h}, \overline{\bar{r}}_{i j}\right) \leq d\left(r_{i j}^{h}, \bar{r}_{i j}\right)$. 
Proof. Expression (22) yields:

$$
d\left(r r_{i j}^{h}, \overline{\bar{r}}_{i j}\right)=\frac{\left|\Delta^{-1}\left(r r_{i j}^{h}\right)-\Delta^{-1}\left(\overline{\bar{r}}_{i j}\right)\right|}{t}
$$

Applying Lemma 1, we have:

$$
d\left(r r_{i j}^{h}, \overline{\bar{r}}_{i j}\right)=\frac{\left|\delta \cdot \Delta^{-1}\left(r_{i j}^{h}\right)+(1-\delta) \cdot \Delta^{-1}\left(\bar{r}_{i j}\right)-\Delta^{-1}\left(\bar{r}_{i j}\right)\right|}{t}=\delta \cdot \frac{\left|\Delta^{-1}\left(r_{i j}^{h}\right)-\Delta^{-1}\left(\bar{r}_{i j}\right)\right|}{t} .
$$

Thus, it is

$$
d\left(r r_{i j}^{h}, \overline{\bar{r}}_{i j}\right)=\delta \cdot d\left(r_{i j}^{h}, \bar{r}_{i j}\right) .
$$

Because $\delta \in[0,1]$ we have that $d\left(r r_{i j}^{h}, \overline{\bar{r}}_{i j}\right) \leq d\left(r_{i j}^{h}, \bar{r}_{i j}\right)$.

Corollary 1. Under the condition of Lemma 1 and for all $\delta<1$, we have:

$$
C I^{h} \leq \overline{C I}^{h}
$$

where $C I^{h}$ is the consensus level computed using the set of set of 2-tuple linguistic decision matrices $\left\{R^{h}=\left(r_{i j}^{h}\right) \mid h=1, \cdots, k\right\}$, while $\overline{C I}^{h}$ is the consensus level computed using the set of set of 2-tuple linguistic decision matrices $\left\{r R^{h}=\left(r_{i j}^{h}\right) \mid h=1, \cdots, k\right\}$.

Proof. From Proposition 1, we have that $d\left(r r_{i j}^{h}-\overline{\bar{r}}_{i j}\right) \leq d\left(r_{i j}^{h}-\bar{r}_{i j}\right)$, and therefore it is

$$
C I^{h}=\frac{1}{m \cdot n} \sum_{i=1}^{m} \sum_{j=1}^{n} C E_{i j}^{h}=\frac{1}{m \cdot n} \sum_{i=1}^{m} \sum_{j=1}^{n}\left(1-d\left(r_{i j}^{h}, \bar{r}_{i j}\right)\right) \leq \frac{1}{m \cdot n} \sum_{i=1}^{m} \sum_{j=1}^{n}\left(1-d\left(r r_{i j}^{h}, \overline{\bar{r}}_{i j}\right)\right)=\overline{C I}^{h}
$$

Consequently, for all $\delta<1$ the feedback mechanism would make the consensus process to converge to a unanimous consensus. The above results are valid when all experts change all their preference values to the feedback recommendation values. Therefore, the production of recommendations to all experts, and their implementation, will guarantee that all expert consensus index values increase. However, in real applications all experts will not receive feedback recommendations, otherwise the computational complexity of the decision making process would increase. Therefore, in the consensus process proposed here only those experts with a consensus level lower than the threshold value will receive recommendations, and if implemented the new collective 2 -tuple linguistic decision matrix will differ from the previous one. In real decision making processes, experts may not accept a recommendation advice if their original preference values require a considerable change in value. The lower the value of $\delta$ the larger the change of preference value in the recommendation. Therefore, an appropriate value of $\delta$ is to be chosen to balance the independence of choice by experts and the consensus level among them. A visual feedback process simulation that generates a graphical simulation of future consensus status if the recommended values were to be implemented is also integrated as part of the visual consensus model here developed, as shown in Figure 9(a) and Figure 9(b). 
Example 8. (Example 2 continuation) Second Consensus Round. Assuming experts $E_{3}$ and $E_{4}$ change their preference values and implement the recommended values, the new collective 2-tuple decision matrix would be

$$
\overline{\bar{R}}=\left(\begin{array}{ccccc}
\left(s_{3}, 0.2\right) & \left(s_{4}, 0.5\right) & \left(s_{4}, 0.5\right) & \left(s_{2}, 0.5\right) & \left(s_{3}, 0\right) \\
\left(s_{5}, 0.1\right) & \left(s_{4},-0.1\right) & \left(s_{4},-0.2\right) & \left(s_{3}, 0.2\right) & \left(s_{3}, 0.2\right) \\
\left(s_{5}, 0.1\right) & \left(s_{2}, 0.3\right) & \left(s_{3}, 0.1\right) & \left(s_{2}, 0.4\right) & \left(s_{3}, 0.5\right) \\
\left(s_{5},-0.2\right) & \left(s_{3}, 0.4\right) & \left(s_{4}, 0.5\right) & \left(s_{2}, 0.4\right) & \left(s_{5},-0.4\right)
\end{array}\right) .
$$

The consensus indexes in this second round would become:

$$
C I^{1}=0.92, C I^{2}=0.90, C I^{3}=0.91, C I^{4}=0.91, C I^{4}=0.92 .
$$

Because all of them are above the consensus threshold value the selection process would be activated.

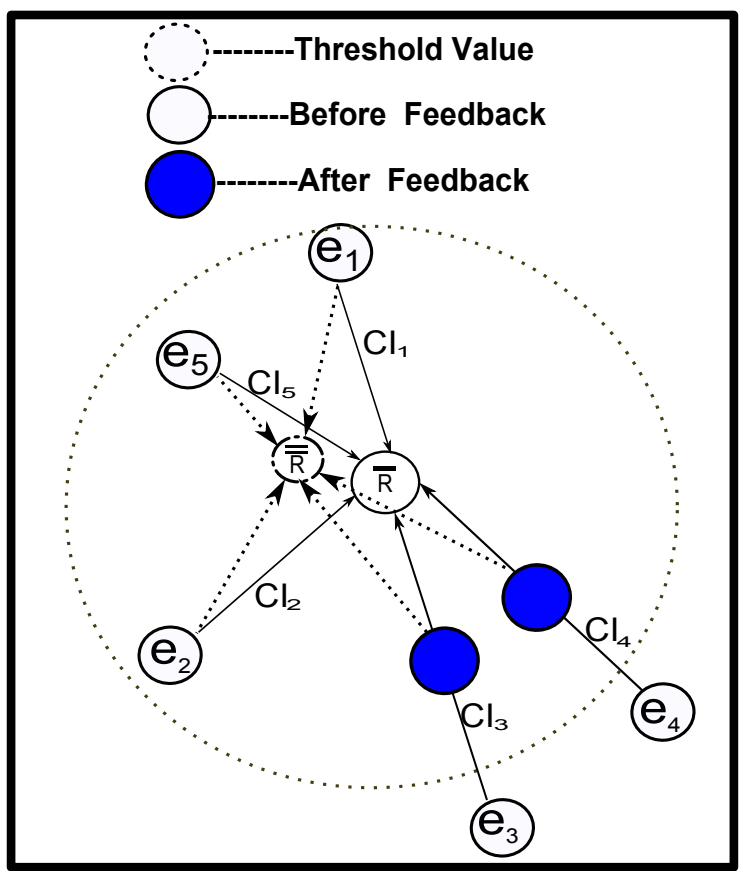

(a) Experts' consensus index on the decision matrix

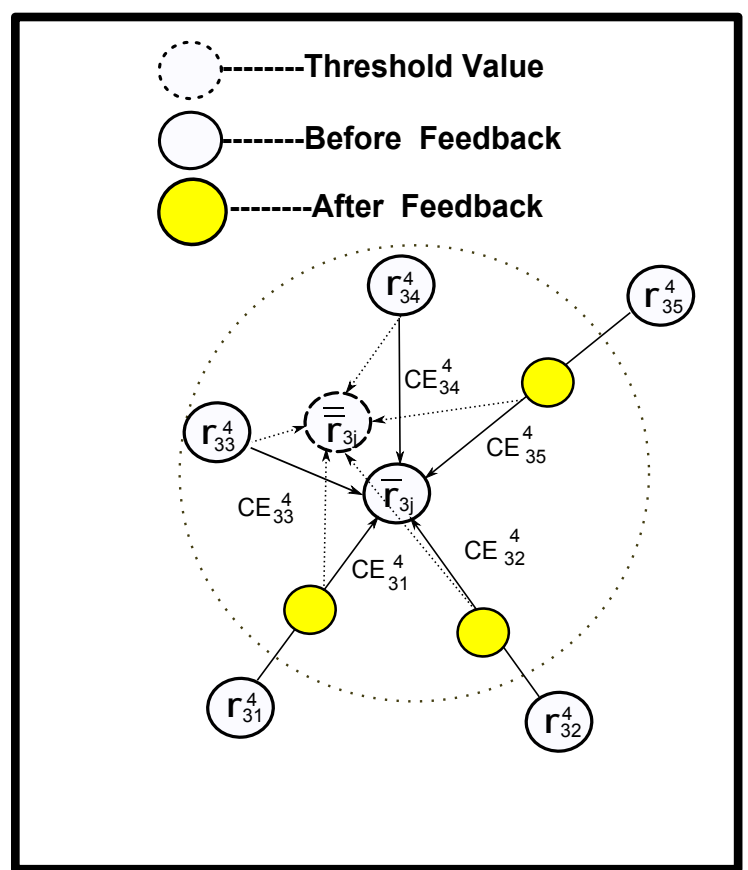

(b) Expert $E_{4}$ consensus index on preferences for alternative $A_{3}$

Figure 9: Visual feedback simulation: consensus levels before and after recommendations implemented by experts

\subsection{Selection Process}

Once the consensus among the group of experts is satisfactory and the collective 2-tuple decision matrix is obtained, we apply the 2-tuple WAA operator to compute the final score for each alternative. Example 9. (Finishing Example 2) Using the criteria weights $\omega=(0.25,0.20,0.15,0.10,0.30)^{T}$, we compute the collective overall evaluation values $\bar{r}_{i}(i=1,2,3,4)$ of the four alternatives:

$$
\bar{r}_{1}=\left(s_{4},-0.4\right), \bar{r}_{2}=\left(s_{4},-0.1\right), \bar{r}_{3}=\left(s_{4},-0.5\right), \bar{r}_{4}=\left(s_{4}, 0.2\right)
$$


The final consensus ranking of alternatives being:

$$
x_{4} \succ x_{2} \succ x_{1} \succ x_{3}
$$

\section{Analysis of the incomplete MCGDM model}

In this article, trust propagating aggregation and visual consensus based decision model for MCGDM with incomplete linguistic information have been investigated. This model has the following main advantages with respect to other decision making models proposed in the literature:

1. It uses the trust relationship in the social network of a group of experts (i.e. the knowledge from other experts) to estimate the unknown preference values in individual decision matrices. Therefore, the developed trust estimation method is completely different from the consistency based estimation methods presented in $[1,12,13,56]$, which rely just in the individual preference values provided in advance by a particular expert (i.e. personal knowledge).

2. It builds a complete network of orthopairs of trust/distrust values from a connected, but not necessarily fully connected, trust network. A trust propagation method to derive some information on an unknown expert by the trusted third parties in an incompletely connected network is investigated and the TS-IOWA operator is developed to aggregate the orthopairs of trust/distrust values obtained from different trust paths by taking into account the risk attitude of experts. Consequently, it is more flexible in its applicability than the K-IOWA operator developed in [48]. Finally, a relative trust score based estimation of unknown linguistic labels as well as an average trust degree based aggregation method for linguistic 2-tuple decision matrices are proposed.

3. It includes a visual consensus model following a top to bottom methodology to provide visually identified discordant opinions, produce recommendations to those experts that are furthest from the group, and visual feedback simulation of future consensus status if experts are to follow recommendations. Furthermore, it guarantees that the production of recommendations to all experts will increase consensus index values, and consequently convergence to consensus. The novelty of the proposed model is that it does not force decision makers to change their opinions, but provide more information to support them to revisit their opinions.

\section{Conclusion}

This article proposes a trust based estimation method and a visual consensus aggregation model for multiple criteria group decision making (MCGDM) with incomplete linguistic information. To do that, a novel trust propagation method is proposed to complete a connected, but not necessarily fully 
connected, trust network. The trust score induced order weighted averaging (TS-IOWA) operator is presented to aggregate the orthopairs of trust/distrust values obtained from different trust paths. The novelty of this operator is that it takes into account the risk attitude of experts. The concepts of relative trust score (RTS) and average trust degree (ATD) are defined. An RTS based estimation and an ATD based aggregation method for the missing preference values are proposed. To help experts achieve the satisfied consensus level, a visual consensus model mechanism is developed and studied, which includes a visual identification to support experts to easily see their consensus position within the group. A recommendation mechanism provides individualised advice to those experts that are identified as contributing less to consensus on how to change their identified element values, which is supplemented with a visual feedback simulation of what would happen if the recommended values were to be implemented by them. Furthermore, it is proved that the feedback mechanism converges to consensus when applied to all experts and all their preferences. In the light of this visual extra information, experts can revisit their evaluations and make changes if considered appropriate in order to achieve a higher consensus level. Consequently, experts are not forced to make changes by the system, rather the system supports them to make free will informed decision regarding possible changes or not of their original preferences.

\section{Acknowledgment}

This work was supported by National Natural Science Foundation of China (NSFC) under the Grant No.71101131 and 71331002, Spanish research projects TIN2010-17876, TIN2013-40658-P, TIC05299, TIC-5991, and the University of Granada 'Strengthening through Short-Visits' (Ref. GENILSSV 2014) programme.

\section{References}

[1] Alonso, S., Chiclana, F., Herrera, F., Herrera-Viedma, E., Alcala-Fdez, J. and Porcel, C. (2008). A consistency-based procedure to estimate missing pairwise preference values. International Journal of Intelligent Systems 23, 155-175.

[2] Alonso, S., Herrera-Viedma, E., Chiclana, F. and Herrera, F. (2009) Individual and social strategies to deal with ignorance situations in multi-person decision making. International Journal of Information Technology and Decision Making 8 (2), 313 - 333.

[3] Alonso, S., Cabrerizo, F.J., Chiclana, F., Herrera, F. and Herrera-Viedma, E. (2009) Group decision-making with incomplete fuzzy linguistic preference relations. International Journal of Intelligent Systems 24 (2), 201-222. 
[4] Alonso, S., Herrera-Viedma, E., Chiclana, F. and Herrera, F. (2010). A Web Based Consensus Support System for Group Decision Making Problems and Incomplete Preferences. Information Sciences, 180 (23), 4477-4495.

[5] Alonso, S, Prez, I.J., Cabrerizo, F.J. and Herrera-Viedma, E. (2013). A Linguistic Consensus Model for Web 2.0 Communities. Applied Soft Computing 13 (1), 149-157.

[6] Cabrerizo, F.J., Moreno, J.M., Prez, V and Herrera-Viedma, E. (2010). Analyzing consensus approaches in fuzzy group decision making: advantages and drawbacks. Soft Computing 14 (5), 451-463.

[7] Cabrerizo, F.J., Prez, I.J. and Herrera-Viedma, E. (2010). Managing the Consensus in Group Decision Making in an Unbalanced Fuzzy Linguistic Context with Incomplete Information. Knowledge-Based Systems 23 (2), 169-181.

[8] Chen, T-Y. (2013). An interactive method for multiple criteria group decision analysis based on interval type-2 fuzzy sets and its application to medical decision making. Fuzzy Optimization and Decision Making, 12 (3), 323-356.

[9] Chen, T-Y. (2014). An ELECTRE-based outranking method for multiple criteria group decision making using interval type-2 fuzzy sets. Information Sciences, 263, 1-21.

[10] Chen T-Y. (2015). The inclusion-based TOPSIS method with interval-valued intuitionistic fuzzy sets for multiple criteria group decision making. Applied Soft Computing, 26, 57-73.

[11] Chiclana, F., Herrera-Viedma, E., Herrera, F. and Alonso, S. (2007). Some induced ordered weighted averaging operators and their use for solving group decision-making problems based on fuzzy preference relations. European Journal of Operational Research 182(1), 383-399.

[12] Chiclana, F., Mata, F., Alonso, S., Herrera-Viedma, E. and Martínez, L. (2008). Integration of a consistency control module within a consensus decision making model. International Journal of Uncertainty, Fuzziness and Knowledge Based Systems 16, 35-53.

[13] Chiclana, F., Herrera-Viedma, E., Alonso, S. and Herrera, F. (2009). Cardinal consistency of reciprocal preference relations: a characterization of multiplicative transitivity. IEEE Transactions on Fuzzy Systems 17(1), 14-23.

[14] Chiclana, F., Tapia-Garcia, J. M., del Moral, M. J. and Herrera-Viedma, E. (2013). A statistical comparative study of different similarity measures of consensus in group decision making. Information Sciences, 221, 110-123.

[15] Chiclana, F. and Zhou, S.M. (2013). Type-reduction of general type-2 fuzzy sets: the type-1 OWA approach. International Journal of Intelligent Systems 28 (5), 505-522. 
[16] Ciucci, D. (2011) Orthopairs: a simple and widely used way to model uncertainty. Fundamenta Informaticae 108 (3-4), 287-304.

[17] Davey, A. and Olson, D. (1998). Multiple criteria decision making models in group decision support. Group Decision and Negotiation, 7(1), 55-75.

[18] De Cock, M. and Pinheiro Da Silva, P.(2006). A many-valued representation and propagation of trust and distrust. Lecture Notes in Computer Science 3849, 108-113.

[19] Dong, YC., Xu, YF., Li, HY. and Feng B. (2010). The OWA-based consensus operator under linguistic representation models using position indexes. European Journal of Operational Research, 203, 455-463.

[20] Dong, YC. and Herrera-Viedma, E. (2015). Consistency-driven automatic methodology to set interval numerical scales of 2-tuple linguistic term sets and its use in the linguistic GDM with preference relation. IEEE Transactions on Cybernetics. doi: 10.1109/TCYB.2014.2336808.

[21] Dong, YC., Li, CC., Xu, YF. and Gu, X. (2015). Consensus-based group decision making under multi-granular unbalanced 2-tuple linguistic preference relations. Group Decision and Negotiation 24, 217-242 (2015).

[22] Eklund, P., Rusinowska, A. and de Swart, H. (2008). A consensus model of political decisionmaking. Annals of Operations Research 158, 5-20.

[23] Fedrizzi, M., Kacprzyk, J. and Zadrożny S. (1988). An interactive multi-user decision support system for consensus reaching processes using fuzzy logic with linguistic quantifiers. Decision Support Systems, 4 (3), 313-327.

[24] Fedrizzi, M., Kacprzyk, J., Owsiński, J.W. and Zadrożny, S. (1994). Consensus reaching via a GDSS with fuzzy majority and clustering of preference profiles. Annals of Operations Research, $51,127-139$.

[25] Hanneman, R. A. and Riddle, M. (2005). Introduction to social network methods. University of California: Riverside.

[26] Herrera, F. and Martínez, L. (2000). A 2-tuple fuzzy linguistic representation model for computing with words. IEEE Transactions on Fuzzy Systems 8(6), 746-752.

[27] Herrera-Viedma, E., Chiclana, F., Herrera, F. and Alonso, S. (2007). Group decision-making model with incomplete fuzzy preference relations based on additive consistency. IEEE Transactions on Systems, Man, and Cybernetics, Part B: Cybernetics 37(1), 176-189. 
[28] Herrera-Viedma, E., Cabrerizo, F.J., Kacprzyk, J. and Pedrycz, W. (2014). A Review of Soft Consensus Models in a Fuzzy Environment. Information Fusion 17, 4-13.

[29] Kacprzyk, J. and Zadrożny, S. (2001). A model for multiperson multicriteria decision making and consensus reaching under fuzzy preferences. Proc. of EUROFUSE Workshop on Preference Modelling and Applications, pp. 79-83.

[30] Kacprzyk, J. and Zadrożny, S. (2003). An Internet-based group decision support system. Management, vol. VII (28), pp. 4-10.

[31] Khalili-Damghani, K. and Sadi-Nezhad, S. (2013). A hybrid fuzzy multiple criteria group decision making approach for sustainable project selection. Applied Soft Computing, 13 (1), 339-352.

[32] Kim, J. K., Choi, S. H., Han, C. H. and Kim, S. H. (1998). An interactive procedure for multiple criteria group decision making with incomplete information. Computers and Operations Research 35, 295-298.

[33] Li, Y. M, and Lai, C. Y. (2014). A social appraisal mechanism for online purchase decision support in the micro-blogosphere. Decision Support Systems 59 190-205.

[34] Malczewski, J. (1996) A GIS-based approach to multiple criteria group decision-making. International Journal of Geographical Information Systems, 10(8), 955-971.

[35] Marakas, G. H. (2003). Decision support systems in the 21th century. Upper Saddle River, NJ: Pearson Education.

[36] Massanet, S. , Riera, J.V., Torrens, J. and Herrera-Viedma, E. (2014) A new linguistic computational model based on discrete fuzzy numbers for computing with words. Information Sciences 258, 277-290.

[37] Mata, F. , Pérez, L. G., Zhou, S.M. and Chiclana, F. (2014). Type-1 OWA methodology to consensus reaching processes in multi-granular linguistic contexts. Knowledge-Based Systems 58, $11-22$.

[38] Martínez, L. and Herrera, F. (2012). An overview on the 2-tuple linguistic model for computing with words in decision making: Extensions, applications and challenges. Information Sciences 207, 1-18.

[39] Morente-Molinera, J.A., Prez, I.J., Ureña, M.R. and Herrera-Viedma, E. (2015). On multigranular fuzzy linguistic modelling in group decision making problems: a systematic review and future trends. Knowledge-Based Systems 74, 49-60. 
[40] Pérez-Asurmendi, P., and Chiclana, F. (2014). Linguistic majorities with difference in support. Applied Soft Computing 18, 196 - 208.

[41] Pérez, I.J., Cabrerizo, F.J. and Herrera-Viedma, E. (2010). A Mobile Decision Support System for Dynamic Group Decision Making Problems. IEEE Transactions on Systems, Man and Cybernetics - Part A: Systems and Humans 40 (6), 1244-1256.

[42] Pérez, I.J., Cabrerizo, F.J., Alonso, S. and Herrera-Viedma, E. (2014). A New Consensus Model for Group Decision Making Problems with Non Homogeneous Experts. IEEE Transactions on Systems, Man, and Cybernetics: Systems 44 (4), 494-498.

[43] Pérez, L. G., Mata, F. and Chiclana, F. (2014). Social network decision making with linguistic trustworthiness based induced OWA operators. International Journal of Intelligent Systems 29 (12), $1117-1137$.

[44] Porcel, C. and Herrera-Viedma, E. (2010). Dealing with Incomplete Information in a Fuzzy Linguistic Recommender System to Disseminate Information in University Digital Libraries. Knowledge-Based Systems 23, 32-39.

[45] Ureña, R., Chiclana, F., Morente, J. A., and Herrera-Viedma, E. (2015). Managing Incomplete Preference Relations in Decision Making: A Review and Future Trends. Information Sciences 302, 14-32 (2015).

[46] Song, F. (2009). Intergroup trust and reciprocity in strategic interactions: Effects of group decision-making mechanisms. Organizational Behavior and Human Decision Processes 108, 164173.

[47] Scott, H. P. (2000). Social Network Analysis: A Handbook. Sage Publications Ltd: London.

[48] Victor, P., Cornelis, C., De Cock, M., and Pinheiro da Silva., P. (2009). Gradual trust and distrust in recommender systems. Fuzzy Sets and Systems 160(10), 1367-1382.

[49] Victor, P., Cornelis, C., De Cock, M., and Herrera-Viedma., E. (2011). Practical aggregation operators for gradual trust and distrust. Fuzzy Sets and Systems 184(1), 126-147.

[50] Wasserman, S. and Faust, K. (2009). Social Network Analysis: Methods and Applications. Cambridge University Press.

[51] Wu, J., Cao, Q. W. and Zhang, J. L. (2010). Some properties of the induced continuous ordered weighted geometric operators in group decision making. Computers and Industrial Engineering 59, 100-106. 
[52] Wu, J. and Chiclana, F. (2012). Non-dominance and attitudinal prioritisation methods for intuitionistic and interval-valued intuitionistic fuzzy preference relations. Expert Systems with Applications 39, 13409-13416.

[53] Wu, J. and Chiclana, F. (2014). Visual information feedback mechanism and attitudinal prioritisation method for group decision making with triangular fuzzy complementary preference relations. Information Sciences 279, 716-736.

[54] Wu, J. and Chiclana, F. (2014). A social network analysis trust-consensus based approach to group decision-making problems with interval-valued fuzzy reciprocal preference relations. KnowledgeBased Systems 59, 97-107.

[55] Wu, J. and Chiclana, F. (2014). A risk attitudinal ranking method for interval-valued intuitionistic fuzzy numbers based on novel score and accuracy expected functions. Applied Soft Computing Volume 22, 272-286.

[56] Wu, J. and Chiclana, F. (2014). Multiplicative consistency of intuitionistic reciprocal preference relations and its application to missing values estimation and consensus building. KnowledgeBased Systems Volume 71, 187-200.

[57] Xu, J. P and Wu, Z. B. (2013). A maximizing consensus approach for alternative selection based on uncertain linguistic preference relations. Computers and Industrial Engineering 64, 999-1008.

[58] Yager, R. R. (1988). On ordered weighted averaging aggregation operators in multicriteria decision making. IEEE Transactions on Systems, Man and Cybernetics 18, 183-190.

[59] Yager, R. R. (1996). Quantifier guided aggregation using OWA operators. International Journal of Intelligent Systems 11, 49-73.

[60] Yager, R. R., and Filev, D. P. (1999). Induced ordered weighted averaging operators. IEEE Transactions on Systems, Man and Cybernetics 29, 141-150.

[61] Yager, R. R., Kacprzyk, J. and Beliakov, G. (2011). Recent developments in the ordered weighted averaging operators: theory and practice. Studies in Fuzziness and Soft Computing. Springer.

[62] Zadeh, L.A. (1975). The concept of a linguistic variable and its application to approximate reasoning-I. Information Sciences 8, 199-249.

[63] Zadeh, L.A. (1975). The concept of a linguistic variable and its application to approximate reasoning-II. Information Sciences 8, 301-357, 1975.

[64] Zhang, BW., Dong, YC. and Xu, YF. (2014). Multiple attribute consensus rules with minimum adjustments to support consensus reaching. Knowledge-based Systems 67, 35-48. 
[65] Zhang, L., Li, T. and Xu, X. (2014). Consensus model for multiple criteria group decision making under intuitionistic fuzzy environment. Knowledge-Based Systems, 57, 127-135

[66] Zhou, S -M., Chiclana, F. , John, R. I. and Garibaldi, J. M. (2008). Type-1 OWA operators for aggregating uncertain information with uncertain weights induced by type-2 linguistic quantifiers. Fuzzy Sets and Systems, 159 (24), 3281-3296. 